\title{
Deconstructing Complexity: Serial Block-Face Electron Microscopic Analysis of the Hippocampal Mossy Fiber Synapse
}

\author{
Scott A. Wilke, ${ }^{1}$ Joseph K. Antonios, ${ }^{1}$ Eric A. Bushong, ${ }^{2}$ Ali Badkoobehi, ${ }^{1}$ Elmar Malek, ${ }^{1}$ Minju Hwang, ${ }^{1}$ \\ Masako Terada, ${ }^{2}$ Mark H. Ellisman, ${ }^{2}$ and Anirvan Ghosh ${ }^{1,3}$ \\ ${ }^{1}$ Neurobiology Section, Division of Biological Sciences and ${ }^{2}$ National Center for Microscopy and Imaging Research, University of California, San Diego, La \\ Jolla, California 92093, and ${ }^{3}$ Neuroscience Discovery and Translation Area, Pharma Research and Development, F. Hoffmann-La Roche, 4070 Basel, \\ Switzerland
}

The hippocampal mossy fiber (MF) terminal is among the largest and most complex synaptic structures in the brain. Our understanding of the development of this morphologically elaborate structure has been limited because of the inability of standard electron microscopy techniques to quickly and accurately reconstruct large volumes of neuropil. Here we use serial block-face electron microscopy (SBEM) to surmount these limitations and investigate the establishment of MF connectivity during mouse postnatal development. Based on volume reconstructions, we find that MF axons initially form bouton-like specializations directly onto dendritic shafts, that dendritic protrusions primarily arise independently of bouton contact sites, and that a dramatic increase in presynaptic and postsynaptic complexity follows the association of MF boutons with CA3 dendritic protrusions. We also identify a transient period of MF bouton filopodial exploration, followed by refinement of sites of synaptic connectivity. These observations enhance our understanding of the development of this highly specialized synapse and illustrate the power of SBEM to resolve details of developing microcircuits at a level not easily attainable with conventional approaches.

\section{Introduction}

The hippocampal mossy fiber (MF) terminal is one of the largest synaptic structures in the brain and is capable of powerfully influencing the spiking activity of the postsynaptic neuron (Henze et al., 2002a,b; Rollenhagen et al., 2007). Each dentate gyrus (DG) granule neuron gives rise to 10-15 MF terminals onto CA3 neurons, whereas each CA3 neuron receives MF input from 50 DG neurons (Amaral et al., 1990; Acsády et al., 1998). A single MF bouton (MFB) can communicate with its postsynaptic partner at up to 37 individual sites of synaptic release (Chicurel and Harris, 1992). This arrangement has led to the characterization of the MF terminal as a "detonator synapse" for its function in sparsely and powerfully activating a particular subset of the CA3 excitatory network (Bischofberger et al., 2006). This same structure also elaborates remarkable filopodial extensions, which provide feedforward inhibition within the CA3 region via synapses onto local interneurons (Acsády et al., 1998; Lawrence and McBain, 2003; Mori et al., 2004; McBain, 2008). The synapses

Received March 31, 2012; revised Sept. 10, 2012; accepted 0ct. 21, 2012.

Author contributions: S.A.W., M.H.E., and A.G. designed research; S.A.W. and E.A.B. performed research; S.A.W., J.K.A., A.B., E.M., M.H., and M.T. analyzed data; S.A.W. and A.G. wrote the paper.

This work was supported by National Center for Research Resources Grant 5P41RR004050 (M.H.E.), National Institutes of Health Grant NS064124 (A.G.), and a research grant from the Gatsby Charitable Trust (A.G.).

Correspondence should be addressed to Anirvan Ghosh, Division of Biology, 1118 Pacific Hall, MC0366, 9500 Gilman Drive, La Jolla, CA 92093-0366. E-mail: aghosh@ucsd.edu.

DOI:10.1523/JNEUROSCI.1600-12.2013

Copyright $\odot 2013$ the authors $\quad 0270-6474 / 13 / 330507-16 \$ 15.00 / 0$ made by MFs play a critical role in gating information transfer in the hippocampus and are considered central to pattern separation and the establishment of spatial memory (Nicoll and Schmitz, 2005; Bischofberger et al., 2006; Leutgeb et al., 2007).

Despite the importance of the MF terminal in hippocampal function, we know relatively little about the structural organization of these synapses because of the challenges associated with electron microscopy (EM)-level reconstruction of complex regional microcircuits. An emerging technology called serial blockface electron microscopy (SBEM) allows the rapid acquisition of large volumes of perfectly aligned, high-resolution ultrastructural data (Denk and Horstmann, 2004; Briggman and Denk, 2006). This groundbreaking technique promises to dramatically increase our understanding of the organization of neural circuits, but its deployment to answer biological questions is still underway (Briggman et al., 2011). Here we demonstrate that SBEM can be driven to resolve ultrastructural features consistent with sites of synaptic contact, whereas the large volumes of perfectly aligned data also enable detailed analysis of local connectivity within CA3 microcircuitry. The MF terminal is ideally suited for this type of analysis because of its large size, multiple release sites, and complex local connectivity. We find that, early in development, MF presynaptic and postsynaptic structures primarily arise independently but associate to form synapses in the second postnatal week, leading to a period of rapid emergence of structural complexity. We also identify previously unappreciated refinement of MF terminal organization, including a fourfold reduction in presynaptic filopodia and a reorganization of sites of 

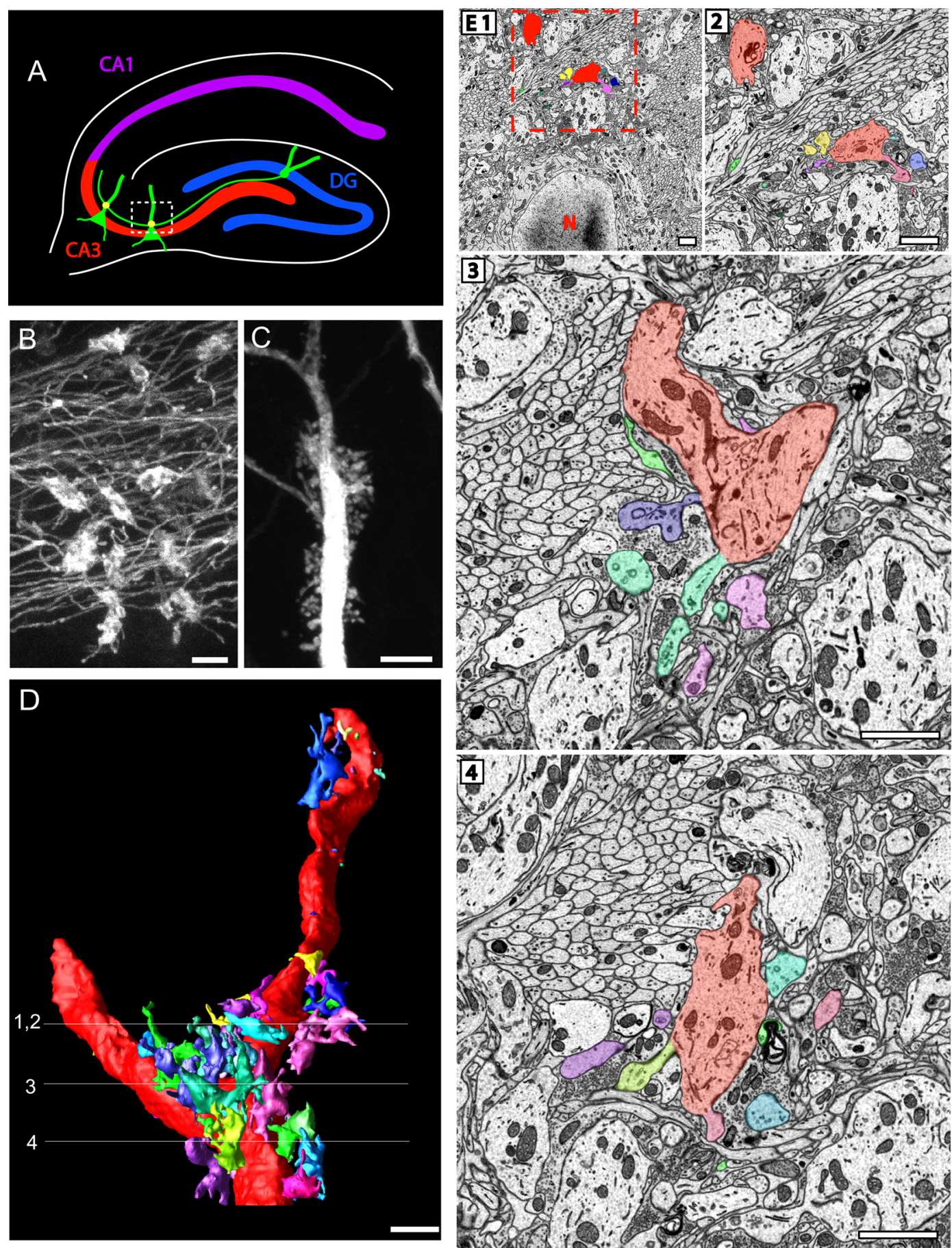

Figure 1. Application of SBEM to the study of hippocampal MF terminals. A, Schematic representation of the hippocampus with the region of SBEM data collection (CA3 SL) indicated (dashed box). B, Dil-labeled MFBs. Scale bar, $5 \mu \mathrm{m}$. C, CA3 neuron with TE spines labeled by intracellular injection of $L Y$ dye in fixed tissue. Scale bar, $5 \mu \mathrm{m}$. $\boldsymbol{D}$, Reconstruction of CA3 neuron apical dendritic shaft (red), with TE spines each in a separate color. Scale bar, $2 \mu \mathrm{m}$. Numbered lines demonstrate planes of SBEM data, which correspond to raw data shown in E1-E4. E1, Full field of view image from SBEM instrument; dashed box is region of interest for subsequent sections in E2-E4. N, Cell nucleus. E2-E4, Raw data images corresponding to planes as indicated in $\boldsymbol{D}$ with contours pseudocolored in correspondence with reconstruction in $\boldsymbol{D}$. Scale bar, $1 \mu \mathrm{m}$.

synaptic contact. These observations enhance our understanding of the development of MF synaptic connectivity and indicate the feasibility of large-scale circuit reconstruction with synaptic resolution.

\section{Materials and Methods}

Fixation and tissue processing for SBEM. All experiments were performed under the University of California, San Diego Animal Care and Use Committee guidelines. C57BL/6J strain of mice (The Jackson Labo- ratory) of either sex, at postnatal day 0 (P0), P7, P14, and P120 (adult) were anesthetized with sodium pentobarbital and then perfused briefly with Ringer's solution containing xylocaine and heparin, followed by $0.15 \mathrm{~m}$ cacodylate buffer containing $2 \%$ paraformaldehyde, $2.5 \%$ glutaraldehyde, and $2 \mathrm{~mm} \mathrm{CaCl}_{2}$. The brain was then dissected free and postfixed in the same fixative solution at $4^{\circ} \mathrm{C}$ for $7 \mathrm{~h}$. Coronal sections, $100 \mu \mathrm{m}$ thick, were cut from the brain in ice-cold $0.15 \mathrm{M}$ cacodylate buffer containing $2 \mathrm{~mm} \mathrm{CaCl}_{2}$ using a vibratome. Slices were then washed in cacodylate buffer for $2 \mathrm{~h}$ at $4^{\circ} \mathrm{C}$ and placed in 
cacodylate buffer containing $2 \% \mathrm{OsO}_{4} / 1.5 \%$ potassium ferrocyanide for $1 \mathrm{~h}$ at room temperature. Slices were washed three times for $5 \mathrm{~min}$ in double-distilled $\mathrm{H}_{2} \mathrm{O}\left(\mathrm{ddH}_{2} \mathrm{O}\right)$ at room temperature before being placed in a filtered solution of $1 \%$ thiocarbohydrazide (EMS) in $\mathrm{ddH}_{2} \mathrm{O}$ for $25 \mathrm{~min}$ at room temperature to allow additional osmium staining. Slices were washed three times in $\mathrm{ddH}_{2} \mathrm{O}$ and then placed in $2 \%$ aqueous $\mathrm{OsO}_{4}$ for an additional $30 \mathrm{~min}$ at room temperature. Finally, slices were washed an additional three times in $\mathrm{ddH}_{2} \mathrm{O}$ and placed in $2 \%$ aqueous uranyl acetate at $4^{\circ} \mathrm{C}$ overnight.

The next day, slices were washed three times in $\mathrm{ddH}_{2} \mathrm{O}$. En bloc lead staining was performed to enhance membrane contrast as described previously (Walton, 1979). A lead aspartate solution was made by dissolving $0.066 \mathrm{~g}$ of lead nitrate in $10 \mathrm{ml}$ of $0.003 \mathrm{M}$ aspartic acid. The $\mathrm{pH}$ was adjusted to 5.5 with $1 \mathrm{~N} \mathrm{KOH}$, and the solution was placed in a $60^{\circ} \mathrm{C}$ oven for $30 \mathrm{~min}$. The lead aspartate solution was filtered, and slices were stained at $60^{\circ} \mathrm{C}$ for $30 \mathrm{~min}$. It was determined that this enhanced osmium staining protocol, combined with en bloc lead staining, was critical for enhancing membrane contrast. Slices were then washed three times in $\mathrm{ddH}_{2} \mathrm{O}$ and then dehydrated in a series of ethanol solutions $(70,90,100$, $100 \%$; $10 \mathrm{~min}$ each, on ice). Slices were then placed in ice-cold dry acetone for $10 \mathrm{~min}$, followed by $10 \mathrm{~min}$ in acetone at room temperature. Slices were then gradually equilibrated with Durcupan Araldite embedding resin by placing in 25\% Durcupan/acetone for $3 \mathrm{~h}, 50 \%$ Durcupan/ acetone overnight, 75\% Durcupan/acetone for $3 \mathrm{~h}$, and 100\% Durcupan overnight. Slices were then flat mounted in fresh 100\% Durcupan and placed in a $60^{\circ} \mathrm{C}$ oven for $48 \mathrm{~h}$ to allow Durcupan polymerization and complete the embedding procedure. The Durcupan Araldite resin recipe was $11.4 \mathrm{~g}$ of component $\mathrm{A}, 10 \mathrm{~g}$ of component $\mathrm{B}, 0.3 \mathrm{~g}$ of component $\mathrm{C}$, and $0.1 \mathrm{ml}$ of component $\mathrm{D}$. Reducing the amount of plasticizer in the formulation of Araldite resin allowed us to embed samples in a harder material and enabled the cutting of sections as thin as $50 \mathrm{~nm}$. We have had success cutting even thinner sections using harder resins, but for these reconstructions, $50 \mathrm{~nm} z$-steps provided sufficient resolution without unnecessarily expanding the size of the final datasets and time of acquisition.

Intracellular labeling of thorny excrescence spines and MFBs. C57BL/6J mice of either sex were perfused briefly with PBS, followed by $4 \%$ paraformaldehyde. Coronal sections, $100 \mu \mathrm{m}$ thick, were cut using a vibratome and stored in PBS on ice. For intracellular injection of Lucifer yellow (LY), penetrating microelectrodes were pulled from standard borosilicate capillary glass with filament $(1.0 \mathrm{~mm}$ outer diameter and $0.58 \mathrm{~mm}$ inner diameter) and backfilled with LY dye (5\%). CA3 neurons were filled via iontophoresis under visual guidance and immunostained after filling with rabbit anti-LY (1:1000). For imaging of MFBs, DiI crystals were placed in the DG of coronal sections as obtained above and incubated in PBS at $37^{\circ} \mathrm{C}$ for 1 week. Thorny excrescence (TE) spines and DiI-labeled MFBs were imaged on a Leica confocal microscope under $63 \times$ magnification with $3 \times$ digital zoom.

Acquisition of SBEM data off 3 View microscope. A small piece of empty Durcupan plastic was glued to the top of an aluminum specimen pin using cyanoacrylate glue. After a region of interest was cut from an embedded tissue section, the tissue block was glued to the empty Durcupan, which acted as a spacer between tissue and metal. Using a glass knife, the tissue block was trimmed to a minimal size $(\sim 0.5 \times 0.5 \mathrm{~mm})$, and the surface was trimmed flat. Silver paint was applied to the specimen so as to ground the Durcupan spacer to the aluminum pin. The pin was then coated with a thin layer of gold-palladium in a sputter coater to further enhance conductivity.

Specimens were imaged on an FEI Quanta FEG SEM equipped with a Gatan 3View SBEM system. For most volumes, imaging was conducted at high vacuum; however, for early developmental time points in which tissue was less dense, the chamber pressure needed to be $10-20 \mathrm{~Pa}$ to prevent charging. The accelerating voltage was $2.0-2.5$ $\mathrm{kV}$, and spot size was $2.0-3.0$. Attempts to image the samples at accelerating voltages of $<2.0 \mathrm{kV}$ resulted in excessively noisy data. The best images were collected at $2.5 \mathrm{kV}$. These beam energies are significantly lower than previous reports of up to $7.5 \mathrm{kV}$ (Denk and Horstmann, 2004). However, we have confirmed that, at $2.5 \mathrm{kV}$, the
Table 1. Quantitative analysis of presynaptic and postsynaptic elements

\begin{tabular}{lccccc}
\hline \multicolumn{7}{c}{$\begin{array}{c}\text { Mean } \pm \text { SD } \\
\text { volume }\left(\mu \mathrm{m}^{3}\right)\end{array}$} & $\begin{array}{l}\text { Mean } \pm \text { SD } \\
\text { Runge }\left(\mu \mathrm{m}^{3}\right)\end{array}$ & $\begin{array}{l}\left(\mu \mathrm{m}^{2}\right) \\
\text { Number }\end{array}$ & Range $\left(\mu \mathrm{m}^{2}\right)$ \\
\hline TE spines & & & & & \\
P0 & 13 & $0.13 \pm 0.14$ & $0.0037-0.546$ & $1.82 \pm 1.26$ & $0.146-4.847$ \\
P7 & 106 & $0.07 \pm 0.25$ & $0.0037-2.139$ & $0.96 \pm 1.78$ & $0.014-13.231$ \\
P14 & 261 & $0.63 \pm 0.91$ & $0.0038-6.371$ & $4.94 \pm 6.94$ & $0.017-44.004$ \\
Adult & 45 & $1.64 \pm 1.40$ & $0.0161-7.271$ & $13.85 \pm 11.10$ & $0.533-56.988$ \\
MFBs & & & & & \\
P0 & 7 & $0.97 \pm 0.38$ & $0.50-1.54$ & $7.70 \pm 2.67$ & $5.37-12.23$ \\
P7 & 39 & $2.27 \pm 2.77$ & $0.12-14.65$ & $13.71 \pm 13.78$ & $2.09-71.37$ \\
P14 & 13 & $9.18 \pm 4.89$ & $6.35-16.52$ & $69.78 \pm 39.29$ & $12.34-133.63$ \\
Adult & 6 & $13.51 \pm 3.03$ & $10.00-18.62$ & $66.53 \pm 14.56$ & $49.07-89.79$ \\
\hline & & & & & \\
\hline
\end{tabular}

excitation depth within the sample extends at least $50 \mathrm{~nm}$, meaning that there was little reason to use $z$-steps smaller than $50 \mathrm{~nm}$ (data not shown). The final lens aperture was $30 \mu \mathrm{m}$, the pixel dwell time was 5-8 $\mu$ s, and the $z$-step size was $50 \mathrm{~nm}$. The diamond knife was oscillated during cutting, and cutting speed was $0.1 \mathrm{~mm} / \mathrm{s}$. All specimens were imaged at $4500 \times$ magnification, with the final pixel size being either 5.2 or $6.3 \mathrm{~nm}$, depending on the raster size. Before each volume was collected, a low magnification $(\sim 500 \times)$ image was collected of the block face to confirm the anatomical location of the volume based on tissue landmarks, such as the DG, CA3, and the stratum lucidum (SL). The SL was recognized as the region of tissue immediately adjacent to CA3, in which apical dendrites were seen to emerge from CA3 somata.

Once a volume was collected, the histograms for the slices throughout the volume stack were normalized to correct for drift in image intensity during acquisition. Digital micrograph files (.dm3) were normalized using Digital Micrograph and then converted to MRC format. The stacks were converted to eight bit, mosaics were stitched, and volumes were manually traced for reconstruction and analysis as below.

Three-dimensional reconstruction and analysis. To analyze these datasets, we used the publicly available software package IMOD, specifically developed for the visualization and analysis of EM datasets in three dimensions (Kremer et al., 1996; http://bio3d.colorado.edu/imod/). Cross-sectional contours were manually traced for consecutive data slices in the $z$ dimension to determine the boundaries of user-defined objects. For some objects, contours were traced in every other data slice in the $z$ dimension. These contour profiles were used for threedimensional volumetric reconstruction of individual presynaptic and postsynaptic elements from which surface area and volume measurements were obtained. Individual elements were given a surface mesh to create models of synaptic components in virtual space (Fig. $1 D, E$ ). For each sample, several dendritic segments with their constituent presynaptic and postsynaptic components were reconstructed (Table 1). Individual TE spines were defined as a protrusion off the dendritic shaft with a common neck. Individual MFBs were defined by the following criteria: (1) an expansion of the axonal shaft, (2) containing high density of synaptic vesicles, and (3) vesicles clustered at point of direct contact with CA3 neuron dendritic shaft or TE spine. MFB filopodia were defined as follows: (1) a protrusion off the MFB, (2) lacking more than scattered vesicles, and (3) extending $>50 \%$ of the width of the main bouton. Synaptic sites were defined as the point of contact between presynaptic and postsynaptic structures that had the following: (1) increased density of synaptic vesicles, (2) some vesicles touching the presynaptic membrane, and (3) an asymmetric thickening of the postsynaptic membrane. Only synaptic sites perpendicular to the plane of sectioning were reconstructed. Synaptic contact surface area estimates are based on the area of the reconstructed presynaptic and postsynaptic membrane covered by the specializations as defined above. Only fully reconstructed TEs and MFBs were included in the analysis, although occasionally MFB filopodial processes extended outside our dataset and were not able to be traced to their ends. 
Quality control. The reconstruction of SBEM data using IMOD software was coordinated by S.A.W. but was completed as a collaborative effort by a five-person reconstruction team. Each team member was instructed in the use of IMOD software by S.A.W. and was initially trained with previously reconstructed datasets to ensure accuracy and consistency. P0 and P7 TE spines were judged to be the least complex to reconstruct, and novice reconstructors started on these before moving on to more complex reconstructions. After completing the training set, reconstructors had $100 \%$ of their work checked by S.A.W. until consistent accuracy was achieved. Spot checks of data were regularly conducted to further ensure accuracy. Finally, a log was kept of all objects traced, which recorded information about the type of structure and whether reconstruction had been completed or was in process. Tools within the IMOD software were used for grouping individual objects hierarchically into completed structures [e.g., completed MF terminal grouped as bouton membrane, filopodia, axon, mitochondria, vesicles, PSDs (postsynaptic densities), and associated spines].

To assess the accuracy and consistency of sectioning thickness by the SBEM diamond knife, we analyzed mitochondrial profiles. Mitochondria are generally circular in transverse cross-section. Therefore, to independently estimate section thickness, we located mitochondria, which had been sectioned longitudinally and took the maximal diameter in $x y$ divided by the number of sections spanned by that mitochondrion in the $z$ dimension. Using this method gave the following mean estimates of section thickness: $\mathrm{P} 0$, $45.4 \mathrm{~nm}$; P7, $50.8 \mathrm{~nm}$; P14, $44.8 \mathrm{~nm}$; and adult, $49.2 \mathrm{~nm}$.

\section{Results}

Application of SBEM to the analysis of MF terminal development

The investigation of particular synaptic subtypes in the hippocampus is facilitated by the fact that specific classes of inputs are laminarly distributed. CA3 neurons of the hippocampus receive MF inputs specifically onto the proximal aspects of their apical dendrites in the hippocampal SL (Fig. 1A). Furthermore, MF terminals can be unambiguously identified based on their unique presynaptic and postsynaptic structural characteristics. MF presynaptic boutons are large and either present as an en passant varicosity or an enlargement off a small terminal side branch with filopodial extensions off the main bouton, labeled here with DiI (Fig. 1B). Postsynaptically, MFBs form synapses onto unique multiheaded complex dendritic spines termed TEs, which are arranged in tight clusters that elude fine-scale analysis at the light level, labeled here by intracellular injection of LY dye (Fig. 1C).
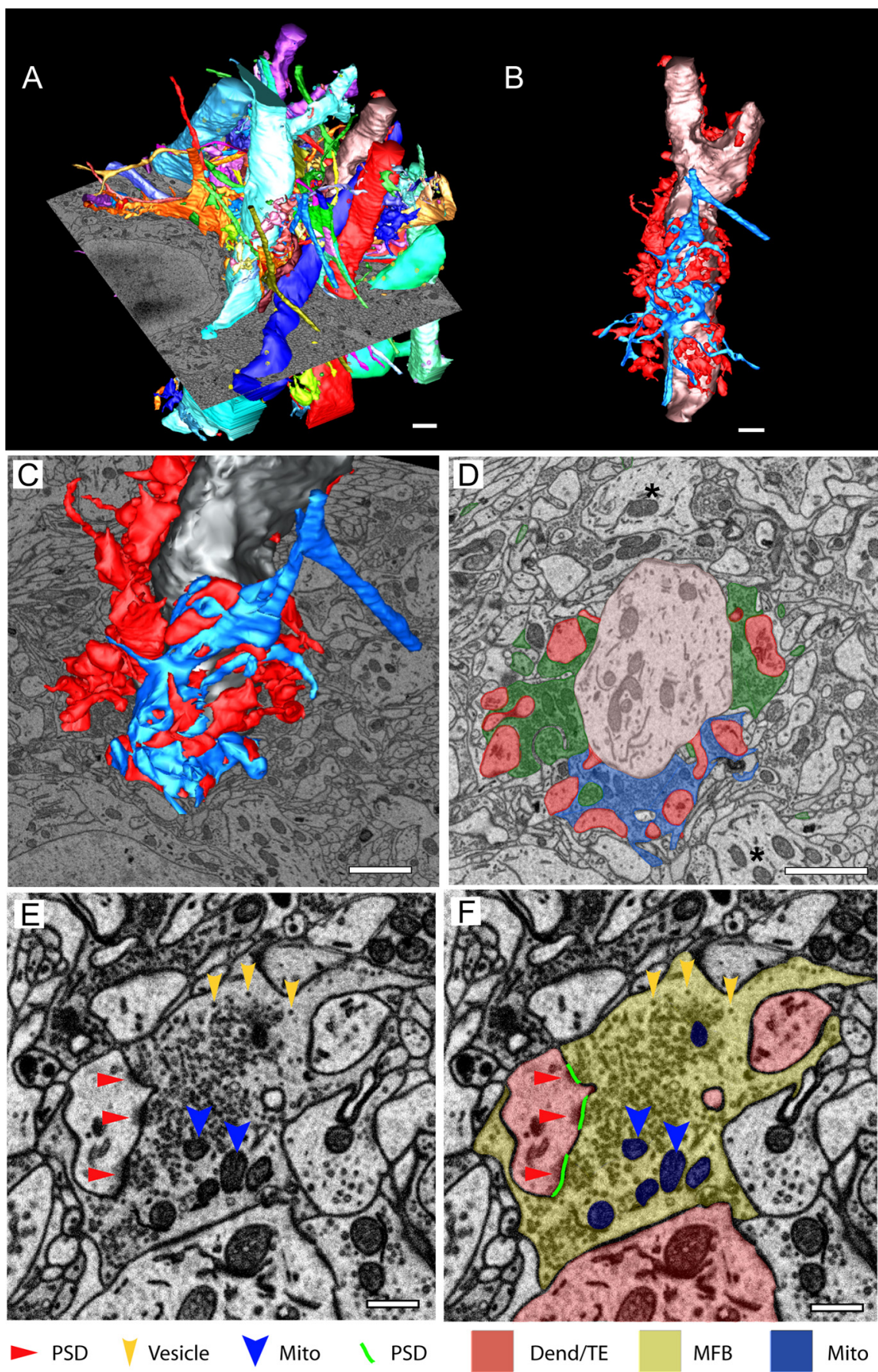

Figure 2. Reconstruction of large volumes of CA3 SL with high resolution at the ultrastructural level. $A$, Representative sample of reconstructed CA3 SL neuropil at P14, showing one plane of raw SBEM data. Data are $\sim 25 \mu \mathrm{m}$ in the $x$ and $y$ dimension and $\sim 20 \mu \mathrm{m}$ in thez dimension. Scale bar, $2 \mu \mathrm{m}$. A number of dendritic shafts are visible along with reconstructed synaptic components. $B$, One CA3 apical shaft reconstruction from $A$, with a single MFB (shaft, pink; spines, red; bouton, blue. Scale bar, $2 \mu \mathrm{m}$. $C$, A portion of the reconstructed dataset shown in $\boldsymbol{B}$, rendered emerging from a single plane of raw data. Scale bar, $2 \mu \mathrm{m}$. $\boldsymbol{D}$, Non-rendered version of the data plane shown in C, with object contours shown in their corresponding colors (shaft, pink; spines, red; bouton, blue; other boutons not shown in Cindicated in green; neighboring shafts, asterisks). Scale bar, $2 \mu \mathrm{m}$. $E$, High-magnification image demonstrating SBEM resolution of ultrastructural features at P14. Arrowheads (red) indicate sites of synaptic release with PSDs apposed to presynaptically clustered vesicles in an MF terminal. Single presynaptic vesicles can be resolved (yellow arrowheads), as well as various organelles (mitochondria, blue arrowheads). $F$, Presynaptic and postsynaptic structures from $\boldsymbol{E}$ are shaded for easier identification: postsynaptic shaft and spine heads (Dend/TE), red; MFB, yellow; PSDs, green; mitochondria (Mito), blue. Scale bars: $E, F, 500 \mathrm{~nm}$.

To investigate MF terminal structures using SBEM, we collected EM volumes from the septal portion of the hippocampus in the CA3 SL, proximal to the hilus of the DG (Fig. 1A, dashed box). The SBEM field for each sample was $\sim 25 \times 25 \mu \mathrm{m}$, 
and we collected EM volumes that extended up to $\sim 20 \mu \mathrm{m}$ in the $z$ dimension composed of sections cut at $50 \mathrm{~nm}$ thickness, resulting in a typical volume of $12,500 \mu \mathrm{m}^{3}$ (Fig. $2 A$ ). These data represent perfectly aligned and continuous blocks of ultrastructural data and can be played as video sequences to provide an overview of the ultrastructural organization of the acquired volume (Movie 1).

To analyze the development of MF terminals, we collected SBEM datasets for the CA3 region of the mouse hippocampus at P0, P7, P14, and adult time points. For each age, we manually reconstructed between one and seven dendritic segments along with their corresponding TE spines and a subset of MFBs from contours drawn in sequential EM cross-sections (Figs. $1 D, E, 2 A$; Table 1). In addition, at each time point, we picked one representative dendritic segment running throughout the entire volume and completely analyzed all of its inputs, for an unbiased assessment of connectivity (see Fig. 6A-L; Table 2).

An example of an isolated CA3 neuron apical dendrite (pink) is shown in Figure $2 B$, with each protrusion emerging from the dendritic shaft traced as a discrete object to facilitate analysis on a per spine basis (red). The reconstruction of a single MFB associated with this dendrite is shown in blue (Fig. 2B). The main bouton was defined as the bulbous vesicle-containing region and was traced separately from the DG axon and filopodial extensions off the bouton (Fig. 2B). Reconstruction of these datasets confirm that volumes were obtained from the SL, with presynaptic and postsynaptic structures closely resembling features of MF terminals visualized at the light level (Fig. $1 B, C$ ). A higher-magnification view of one cross-section of the reconstruction as indicated in Figure $2 C$ is shown in Figure $2 D$ and illustrates how single sections of ultrastructure correspond to reconstructions. Distinct MFBs (green and blue profiles) associated with the dendritic shaft and TEs (red) can be clearly distinguished. Furthermore, individual PSDs were visible in SBEM images, as well as sites of presynaptic vesicle clustering at active zones (Fig. $2 E, F$, red arrowheads and green lines). Within MF presynaptic terminals (yellow), individual vesicles could be resolved (yellow arrowheads), as well as mitochondria (blue with blue arrowheads) and other subcellular structures (Fig. 2E,F).

\section{Maturation of the giant MF presynaptic bouton}

Mature MFBs are large and complex structures, packed with synaptic vesicles (Chicurel and Harris, 1992; Rollenhagen et al., 2007). As early as P0, MF axons clustered vesicles at sites of contact with the dendritic shaft and often had a single mitochondrion associated with the vesicle pool (Fig. 3A,B,G; Table 2). Across development, there was an increase in the size of vesicle pools and the number of associated mitochondria that parallels the growth of individual MFBs (Fig. 3G-J; Table 2). Analysis of MFB morphology at different developmental stages revealed a dramatic increase in MFB size and complexity between P7 and P14, which corresponds to functional maturation of these synaptic structures (Marchal and Mulle, 2004) (Figs. 3 H, I, 4B, C). At $\mathrm{P} 0$, axons rarely had clear bouton-like enlargements but frequently clustered vesicles at their point of contact with the dendritic shaft (Figs. $3 A, B, G, 4 A, E$ ). By P7, axons regularly exhibited varicosities with large numbers of vesicles clustered at sites of contact (Figs. 3C-E, $H, 4 B, E$ ). A single filopodial protrusion often emerged from the main MFB enlargement at this stage of development and sometimes ended in a growth-cone-like structure (Fig. $4 B$, asterisk).

Between P7 and P14, MFBs increase in volume nearly fivefold but maintain a high surface area/volume ratio and seem to ooze

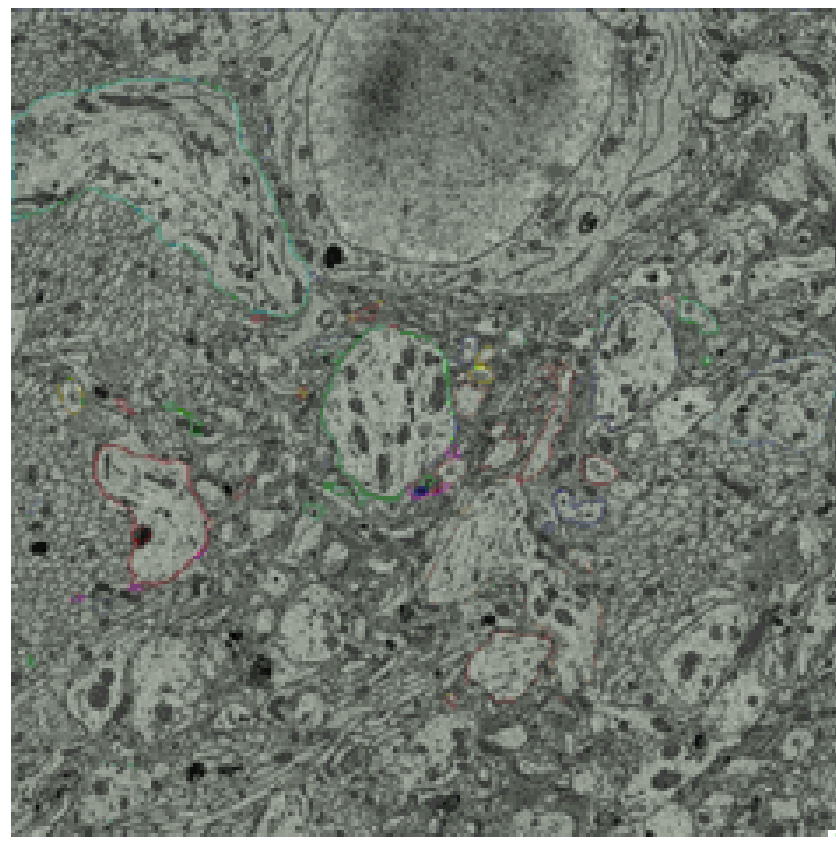

Movie 1. Example SBEM dataset from P14SL with reconstructed objects indicated by colored contours. Movie scans up and down through serial sections in the z-dimension for a part of this data set.

down the dendritic shaft in and out of emerging TE spines (Fig. $4 C, E$; Table 1). By this age, boutons are already nearly 100 -fold larger than varicosities formed by other axons in the hippocampus, which average $\sim 0.125 \mu \mathrm{m}^{3}$ in volume (Harris and Stevens, 1989; Shepherd and Harris, 1998). In addition, there was a dramatic increase in bouton complexity between P7 and P14, with individual boutons elaborating as many as 23 separate filopodial extensions (Fig. 4C,F; Table 2).

Finally, although adult MFBs had a similar surface area to P14 MFBs (Table 1), they had a greater density of synaptic vesicles and were considerably larger and more uniform in shape (Figs. 3I-L, $4 D, E$; Table 1). Interestingly, adult MFBs were significantly less complex than at P14, indicating that the high numbers of filopodial extensions at P14 are a transient developmental phenomenon and may be important for synapse formation (Fig. 4D,F; Table 2).

\section{Emergence of the postsynaptic TE}

The MF terminal transmits information from DG axons to CA3 dendrites via a complex postsynaptic dendritic spine called the TE. MF-evoked AMPA receptor-mediated currents are first detected at approximately $\mathrm{P} 3$ and increase in amplitude across the first 3 postnatal weeks (Marchal and Mulle, 2004). Mirroring this functional maturation, we found that TE spines also dramatically increase in volume with a concomitant increase in complexity during this developmental period (Fig. 5; Table 1). At P0, dendritic protrusions arising from CA3 neurons in the SL were small and only rarely observed (Figs. $5 A, E, 6 A$; Table 2; Movie 2). By P7, dendritic protrusions were observed much more frequently (Fig. 6B; Table 2; Movie 3), and, in contrast to the relatively uniform MFBs at this age, developing TEs were highly variable in morphology (Fig. 5B,F; Table 1). P7 dendritic spines were small "finger-like" protrusions, long and occasionally branched filopodial extensions, or, in rare cases, bulbous with multiple compartments and extensions (Fig. 5B). 
Table 2. Quantitative analysis of connectivity for completely reconstructed dendritic segments

\begin{tabular}{|c|c|c|c|c|}
\hline Characteristics & P0 & P7 & P14 & Adult \\
\hline Dendritic length ( $\mu \mathrm{m})$ & 34.4 & 36.2 & 32.5 & 30.1 \\
\hline Number of TE spines & 2 & 37 & 78 & 46 \\
\hline Density of TE spines (TE/ $\mu \mathrm{m})$ & 0.06 & 1.02 & 2.40 & 1.53 \\
\hline Number of MF inputs & 6 & 19 & 18 & 19 \\
\hline Density of MF inputs (MFB/ $\mu \mathrm{m})$ & 0.17 & 0.53 & 0.55 & 0.63 \\
\hline Mean number of TEs per MFB \pm SD & $0.17 \pm 0.41$ & $0.89 \pm 1.24$ & $3.72 \pm 3.01$ & $2.42 \pm 1.22$ \\
\hline Range & $0-1$ & $0-4$ & $0-10$ & $1-5$ \\
\hline Mean number of filopodia per MFB \pm SD & 0 & $0.12 \pm 0.33$ & $9.43 \pm 9.11$ & $2.33 \pm 2.30$ \\
\hline Range & $\mathrm{N} / \mathrm{A}$ & $0-1$ & $1-23$ & $0-6$ \\
\hline TEs associated with MFB & $50 \%$ & $45.9 \%$ & $85.9 \%$ & $98 \%$ \\
\hline MFBs associated with TE & $17 \%$ & $47 \%$ & $83 \%$ & $100 \%$ \\
\hline Mean volume of TEs associated with MFB $\left(\mu \mathrm{m}^{3}\right)$ & 0.07 & $0.17 \pm 0.51$ & $0.91 \pm 0.79$ & $1.62 \pm 1.21$ \\
\hline Mean volume of TEs not associated with MFB $\left(\mu \mathrm{m}^{3}\right)$ & 0.55 & $0.01 \pm 0.01$ & $0.01 \pm 0.01$ & 0.02 \\
\hline Mean volume of MFBs associated with TE $\left(\mu \mathrm{m}^{3}\right)$ & 0.82 & $1.11 \pm 0.95$ & $10.72 \pm 5.29$ & $13.51 \pm 3.03$ \\
\hline Mean volume of MFBs not associated with TE $\left(\mu \mathrm{m}^{3}\right)$ & $0.73 \pm 1.72$ & $0.85 \pm 0.60$ & $0.64 \pm 0.49$ & $\mathrm{~N} / \mathrm{A}$ \\
\hline Mean number of mitochondria per MFB \pm SEM & $1.29 \pm 0.97$ & $1.62 \pm 0.27$ & $10.00 \pm 2.64$ & $24.00 \pm 2.16$ \\
\hline Range & $0-7$ & $1-4$ & $1-23$ & $18-28$ \\
\hline Mean mitochondria volume/MFB volume \pm SEM (\%) & $6.49 \pm 5.75 \%$ & $4.58 \pm 1.48 \%$ & $5.15 \pm 0.91 \%$ & $6.93 \pm 0.69 \%$ \\
\hline Range & $0.0-21.1 \%$ & $0.23-20.54 \%$ & $0.66-10.05 \%$ & $4.96-8.18 \%$ \\
\hline
\end{tabular}

Between P7 and P14, there was an explosive growth of dendritic protrusions and a dramatic increase in spine density and mean volume (Figs. 5C,G, 6C; Table 2; Movie 4). Volumes of individual spines varied considerably at P14, with the majority of spines falling under $0.2 \mu \mathrm{m}^{3}$ and having a single head, but with some spines as large $6.37 \mu \mathrm{m}^{3}$ and exhibiting an extremely complex, multi-compartmental morphology (Fig. $5 C, G$ ). This variability strongly suggests that, even at P14, the process of TE spinogenesis is still very active and even the largest spines had morphologies, suggestive of active remodeling with many spinule-like processes emerging from existing spine heads (Fig. $5 C$, arrowheads).

Finally, mature adult spines were even larger, having a mean volume nearly three times that observed at P14 (Fig. $5 D, H$; Table 1; Movie 5). Although adult TE morphology was more uniform than at developmental time points, TEs were still highly variable in size and shape, with the majority of spines falling between 0.2 and $2.0 \mu \mathrm{m}^{3}$, but with spines frequently exceeding a volume of $5.0 \mu \mathrm{m}^{3}$ (Fig. $5 D, H$ ). Mature TE spines appear like bunches of grapes emerging from a common neck. Adult TE spines frequently had a small central stalk from which large, round and uniform spine heads emerged (Fig. 5D). Notably, we found one instance of a small filopodial process emerging from the dendritic shaft (data not shown), and TE spines still frequently exhibited spinule structures emerging from existing spine heads (Fig. 5D, arrowheads), consistent with some degree of remodeling in the adult as has been described previously (Galimberti et al., 2006; Toni et al., 2008).

\section{Structural relationships between dendritic and axonal elements at the nascent MF synapse}

Reconstruction of EM data provides a unique opportunity to explore the relationship between the precursors of presynaptic and postsynaptic elements during the process of circuit development. Previous studies have suggested that presynaptic development at the MF synapse precedes postsynaptic development and that MF contact may initiate outgrowth of TE spines in vitro (Amaral and Dent, 1981; Dailey et al., 1994; Robain et al., 1994). This is in contrast to other cortical synapses, in which spine growth frequently precedes synapse formation and filopodial structures often do not have a presynaptic partner (Knott et al.,
2006; Arellano et al., 2007; De Roo et al., 2008). Analysis of classical spines supports a model in which dendritic filopodia actively seek out presynaptic partners before transitioning to a mature spine (Ziv and Smith, 1996; Nimchinsky et al., 2002), although a significant number of synapses appear to form on dendritic shafts (Sorra and Harris, 2000).

Our data indicate that MFBs mature before TE spines (Fig. 6). At $\mathrm{P} 0$, vesicles often clustered at sites of axon-dendrite contact during a period notable for sparse dendritic protrusions (Figs. $3 A, B, G, 4 A, 6 I$; Table 2). By P7, protrusions more frequently associate with boutons packed with vesicles and mitochondria, presumably capable of neurotransmitter release (Figs. $3 E, H, 6 J$ ). In most cases, these protrusions were small and grew out around the periphery of the MFB, whereas vesicle pools were associated with the dendritic shaft (Fig. 3C-E).

Although some dendritic protrusions and axonal varicosities are in association by P7, the majority of dendritic and axonal specializations were not in physical contact with each other at these early stages (Figs. 3C, D, F, 6O; Table 2). For example, at P7, $\sim 50 \%$ of MFBs were not in direct contact with spines, and similarly $\sim 50 \%$ of postsynaptic protrusions were not in contact with vesicle-containing boutons (Figs. 3C, D, F, 6O; Table 2). By P14, only $20 \%$ of MFBs were not in direct contact with spines, and finally, in the mature circuit, no MFBs were found that did not clearly exhibit synapses onto TE spines and only one dendritic filopodial protrusion was identified that did not contact an MFB (Fig. 6O; Table 2). These data indicate that the presence of an MF axonal specialization is not necessary for the existence of an immature dendritic protrusion.

Contact between axonal and dendritic specializations appears to be required for TE maturation because protrusions associated with an MFB were significantly larger than those that were not (Table 2). A limited number of glial cells were reconstructed at P7 and P14 to assess their interaction with axonal and dendritic specializations during synapse formation (Fig. $7 A, B$ ). Glial processes were infrequently identified in $\mathrm{P} 0$ samples and are extraordinarily complex and time-consuming to reconstruct in adult samples. Although a comprehensive analysis of glial development with respect to synapse formation was beyond the scope of this study, some observations are noteworthy. Glial cells were numerous at P7 with long, highly ramified processes present at sites of 

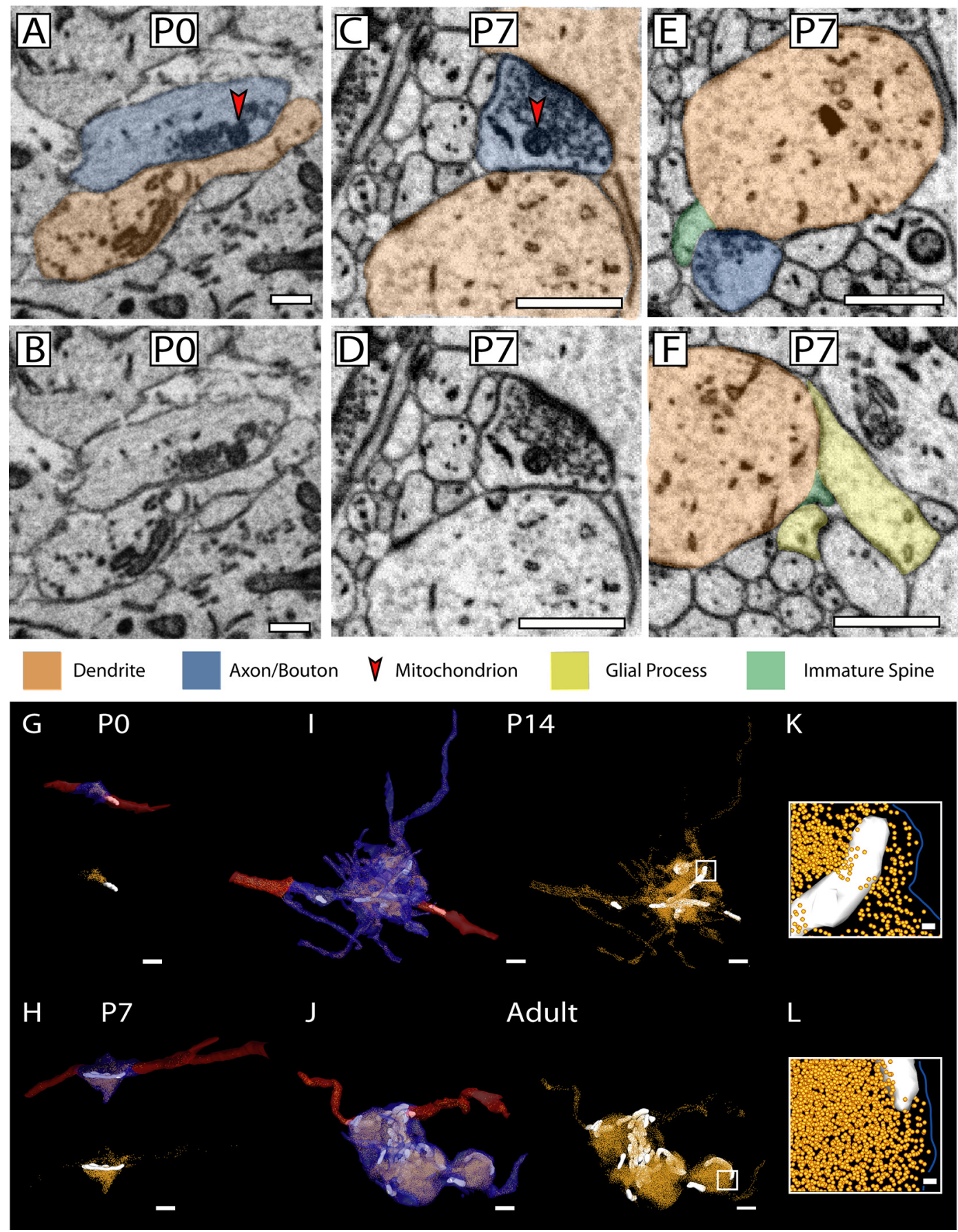

Figure 3. Synapse initiation and development of bouton ultrastructure. $A, P 0$ : early vesicle clustering at site of axonal contact with CA3 dendrite. $\boldsymbol{B}$, Micrograph from $\boldsymbol{A}$ without pseudocoloring. C, P7: early MFB contacting CA3 dendrite in the absence of immature spine. D, Micrograph from C without pseudocoloring. E, P7: early association of axonal and dendritic specializations. F, P7: early dendritic protrusion emerging in contact with glial processes. G, P0 bouton with reconstructed ultrastructure. $\boldsymbol{H}$, P7 bouton with reconstructed ultrastructure. $\boldsymbol{I}$, P14 bouton with reconstructed ultrastructure. J, Adult bouton with reconstructed ultrastructure. $\boldsymbol{K}$, Magnified view of vesicle density from I. L, Magnified view of vesicle density from J. Structure color code: axon, red; bouton, blue; vesicles, orange; mitochondria, white; TE spines and spine precursors, green; glial processes, yellow; dendrite shaft, orange. Arrowheads in $A$ and $C$ indicate mitochondrial profiles. Scale bars: $1 \mu \mathrm{m}$; insets $K$ and $\boldsymbol{L}, 10 \mathrm{~nm}$.

synaptogenesis (Fig. 7 $A, C, D$ ). Interestingly, in numerous instances in P7 and P14 samples, small dendritic protrusions not associated with axonal structures were only in contact with glial processes (Figs. 3F, 7D). Glial cells at P7 also appeared to be present at the sites of initial contact between dendritic protrusions and unspecialized axonal segments (Fig. 7C). This is consistent with the possibility of glial cells actively orchestrating synapse formation, as has been suggested previously (for review, see Ullian et al., 2004). By P14, glial cells began to take on more mature spongiform morphologies with processes in and around early synaptic sites (Fig. $7 B, E, G$ ), whereas adult glial processes often appeared to surround and shield adjacent MF terminals (Fig. $7 F, H$ ).

Consistent with previous studies, we found that a single MFB can contact multiple independent spines emerging from the CA3 neuron dendrite (Amaral and Dent, 1981; Chicurel and Harris, 

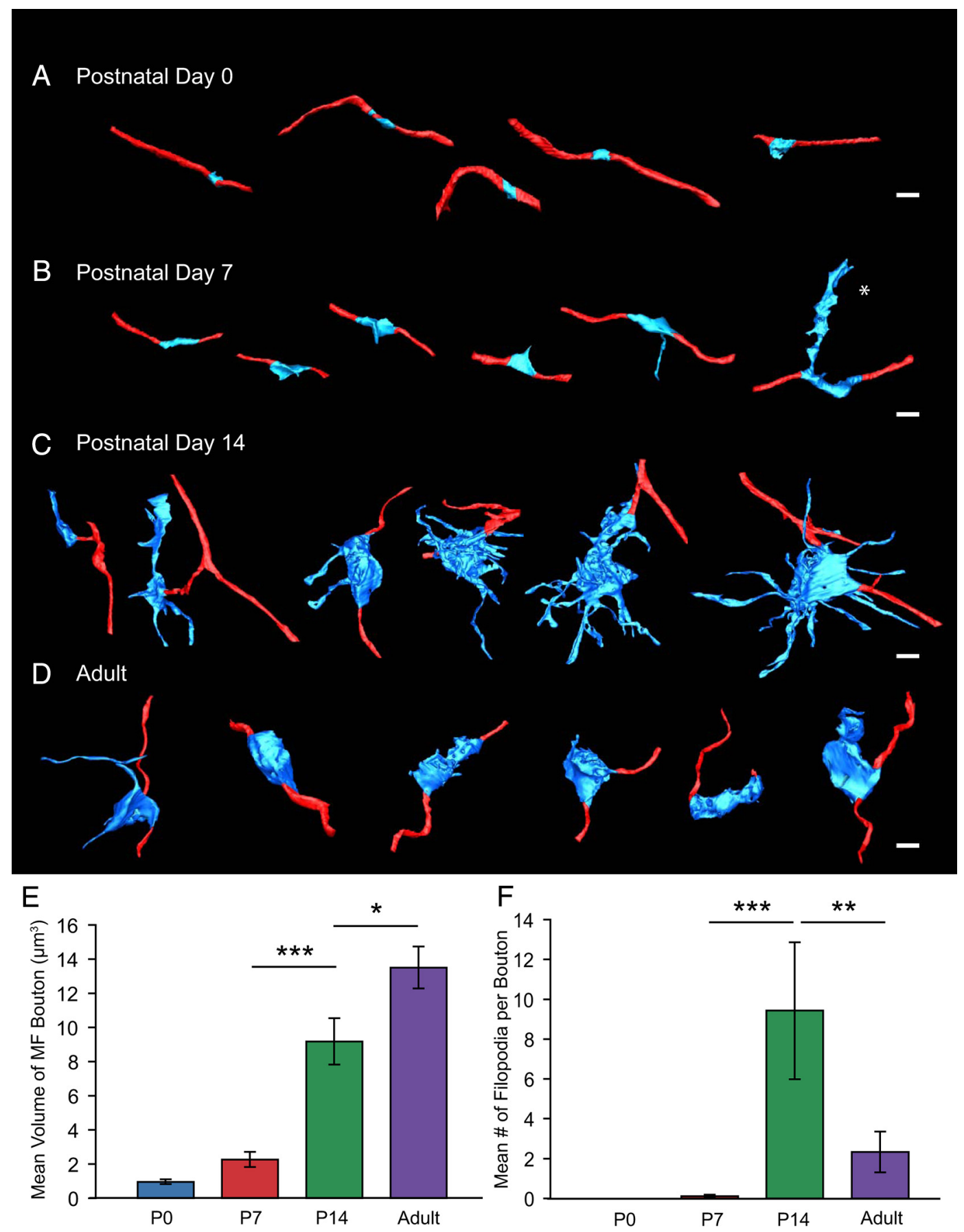

Figure 4. Maturation of MF axonal specializations. MF axons (red) with boutons (regions of vesicle accumulation; blue) were reconstructed from SL samples. $\boldsymbol{A}-\boldsymbol{D}$, Representative MF terminals and axons from P0 (A), P7 (B), P14 (C), and adult (D) mice. E, Quantification of the mean volume of the vesicle-containing portion of MFBs excluding volume of filopodial protrusions for each age. $\boldsymbol{F}$, Quantification of the mean number of filopodial extensions per bouton for each age. Scale bars, $2 \mu \mathrm{m} .{ }^{*} p<0.05,{ }^{* *} p<0.01,{ }^{* * *} p<.001$ by ANOVA. Error bars represent \pm SEM.

1992). At P0 and P7, the majority of MFBs did not contact any dendritic protrusions (83 and 53\%, respectively) (Fig. 6M-O; Table 2). However, by P14, MFBs contacted many more emerging TE spines (only 17\% of MFBs unassociated) (Fig. 6N, O; Table 2). Interestingly, mature adult MFBs contacted fewer individual TE spines than at P14 (Fig. 6M; Table 2), suggesting that there is refinement of presynaptic and postsynaptic association during later development. For MFBs within each developmental stage except $\mathrm{P} 0$, there was a direct relationship between bouton volume and the number of spines contacted (Fig. 6N). At P14, we also identified instances in which two presynaptic bou- tons synapse onto the same TE spine (Fig. $8 A-D$ ) and also in which a single MFB synapses onto TE spines from two separate dendrites (Fig. 9A-E). Similar arrangements were not observed in the adult, consistent with the possibility that such arrangements represent a transient stage in circuit formation.

\section{Development and refinement of sites of synaptic contact}

A major technical advance in our use of SBEM for ultrastructural analysis is the ability to visualize synaptic vesicles clustered at axonal sites opposed to PSDs, representing sites of MF neurotransmission (Fig. 2E,F). To investigate the structural 

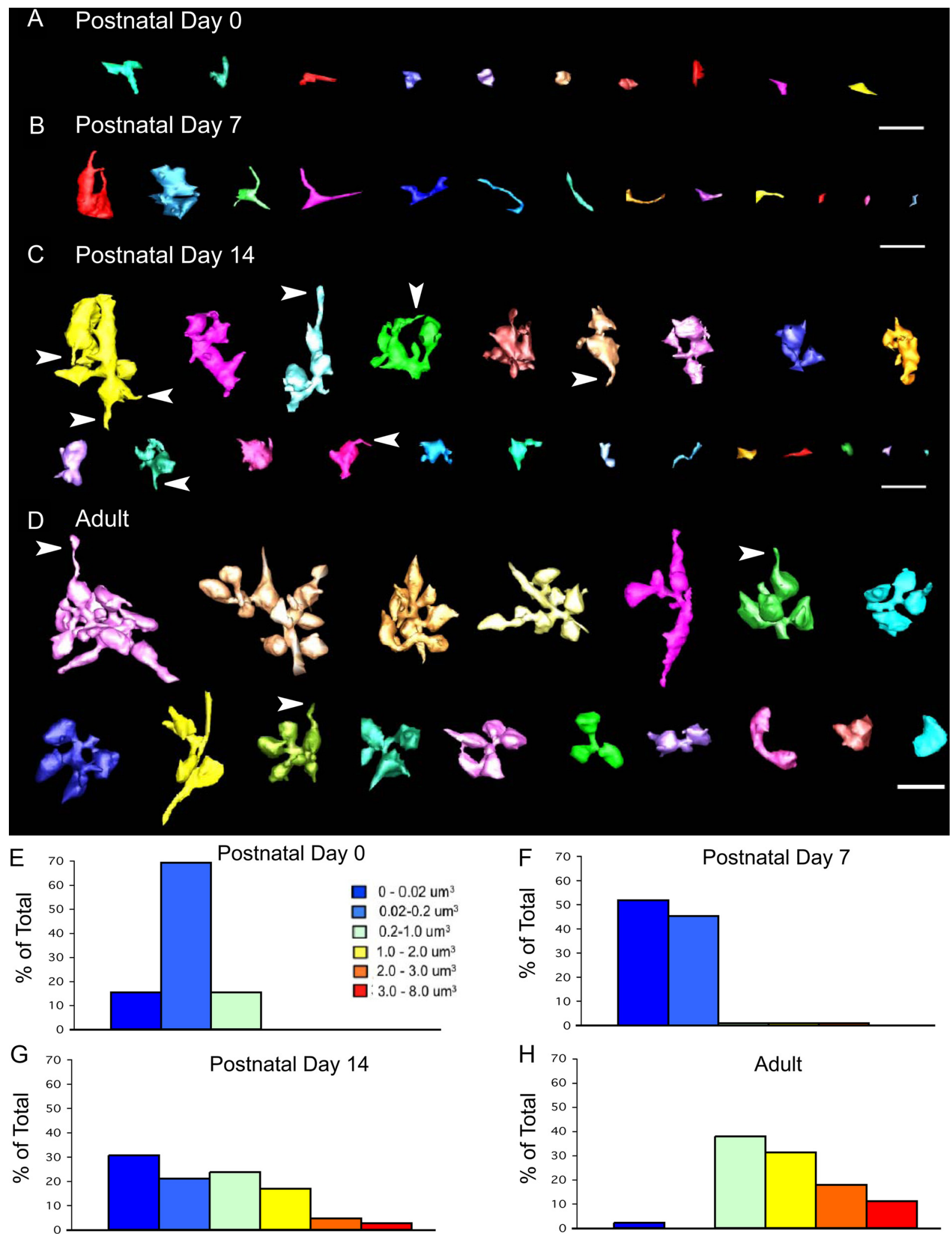

Figure 5. Analysis of the maturation of CA3 dendrite TE structure. Postsynaptic protrusions with a common neck were reconstructed as a single object for analysis. $A-D$, Representative TE spines from P0 (A), P7 (B), P14 (C), and adult (D) mice. $\boldsymbol{E}-\boldsymbol{H}$, TE spine volumes were quantified for each age. Histograms of binned spine volumes from P0 (E), P7 (F), P14 (G), and adult $(\boldsymbol{H})$ mice are displayed. Scale bars, $2 \mu \mathrm{m}$.

maturation of neurotransmission and correlate this process with larger-scale morphological development of presynaptic and postsynaptic structures, we reconstructed the fullcomplement synaptic contacts for TE spines at P7, P14, and adult time points (Fig. $10 A-C$ ). We found that a single TE spine typically only contacts one MFB, but the number of synaptic contacts scales proportionally with the volume of the TE spine (Fig. 10D). 

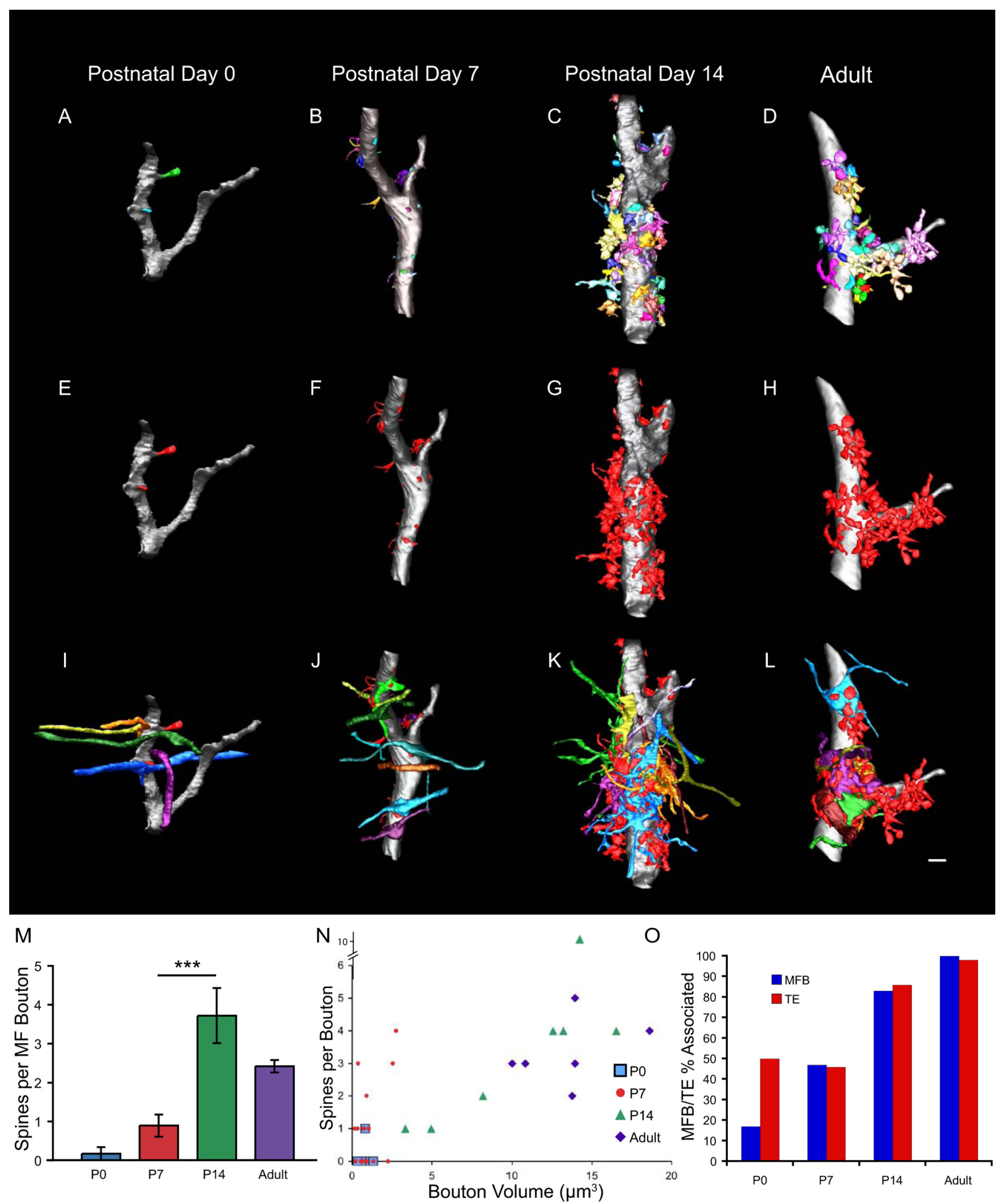

Figure 6. Structural relationships between axonal and dendritic elements demonstrated by reconstruction of large segments of CA3 dendrite with inputs. $A-D$, Reconstruction of dendritic structure in which dendritic shafts are indicated in silver, and each dendritic protrusion is indicated in a separate color for P0, P7, P14, and adult (P120). E-H, Same reconstructions shown in $\mathbf{A}-\mathbf{D}$, with dendritic protrusions indicated in red. $\boldsymbol{I}-\boldsymbol{L}$, Same dendritic reconstructions shown in $\boldsymbol{E}-\boldsymbol{H}$ but with a subset of the reconstructed axons and MFBs that form contacts onto that neuron, each shown in a separate color. $M$, Quantification of mean number of TE spines contacted per MFB for each age. $\boldsymbol{N}$, Plots of the association between the volume of individual MFBs and the number of TE spines, which they contact at each age. $\mathbf{0}$, Quantification of the percentage of MFBs at each age, which are associated with a dendritic protrusion, and the percentage of TE spines, which are associated with a MFB at each age. Scale bar, $2 \mu \mathrm{m} .{ }^{* * *} p<0.001$, by $t$ test. 


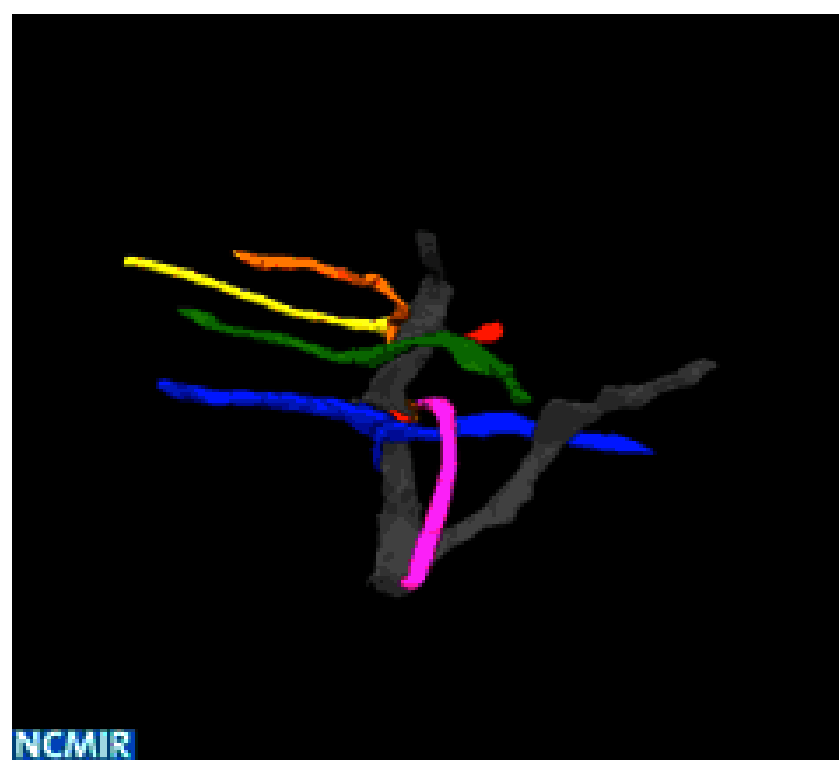

Movie 2. Example PO SBEM reconstruction. CA3 dendritic shaft (silver) is shown with full complement of dendritic protrusions (red), followed by sequential appearance of five contacting MF axons (remaining colors). Not all MF inputs are shown.

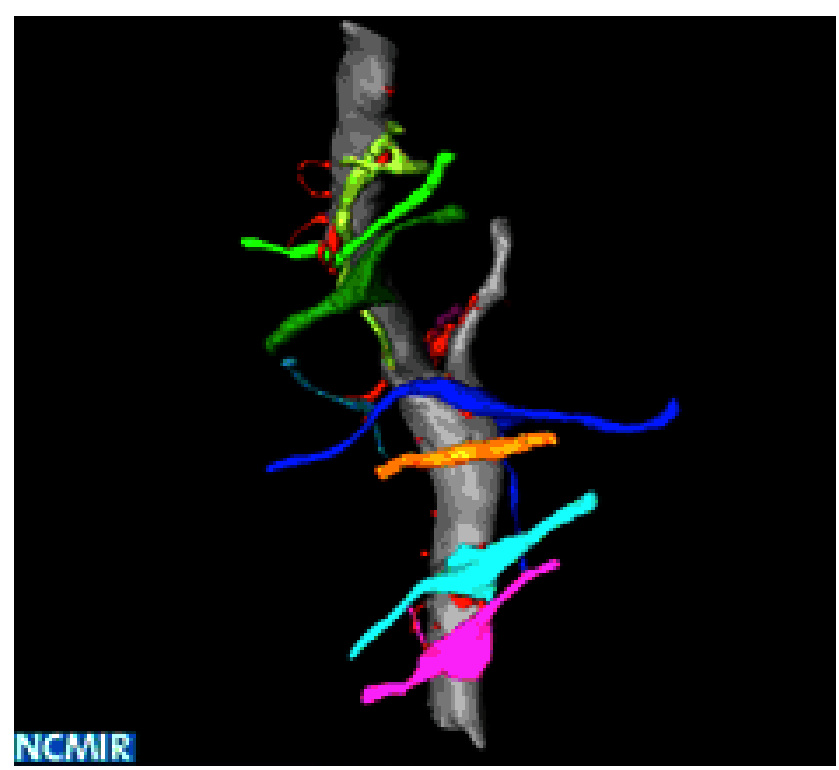

Movie 3. Example P7 SBEM reconstruction. CA3 dendritic shaft (silver) is shown with full complement of immature dendritic spines (red), followed by sequential appearance of nine contacting MF axons/boutons (remaining colors). Not all MF inputs are shown.

At P7, the vesicles of MFBs were usually clustered adjacent to the dendritic shaft, even when the MFB was also in contact with an immature TE spine (Fig. 3C-E). Only rarely did we identify synaptic contacts onto immature spines (Fig. 10A; data not shown). By P14, most TE spines had developed multiple synaptic contacts of varying shapes and sizes, although $31 \%$ still lacked any synapses (Fig. 10B). Finally, in the mature MF circuitry of adult animals, virtually all TE spines had at least one synaptic contact, with $85 \%$ having between two and six such sites (Fig. 10C). At both P14 and adult time points, synaptic contacts could be quite variable in morphology, with many having typical "patch-like" shapes, whereas others were perforated or even "ribbon-like" in distribution (Fig. 10B,C). Although synapse morphology was similar between P14 and adult time points, mature synaptic con-

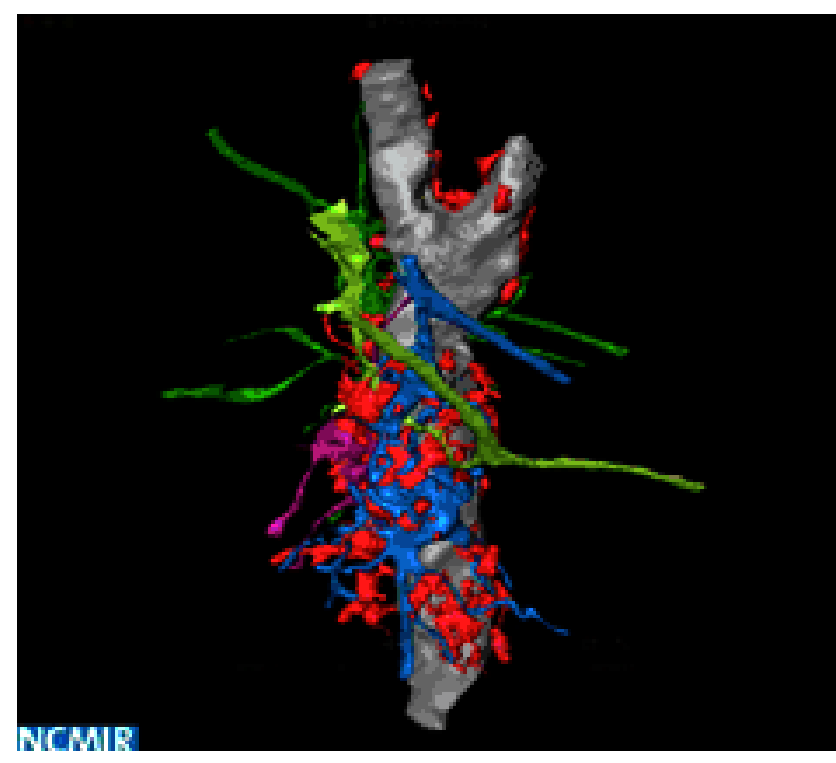

Movie 4. Example P14 SBEM reconstruction. CA3 dendritic shaft (silver) is shown with full complement of dendritic spines (initially shown in separate colors and then turn to red), followed by sequential appearance of four contacting MF axons/boutons (remaining colors). Not all MF inputs are shown.

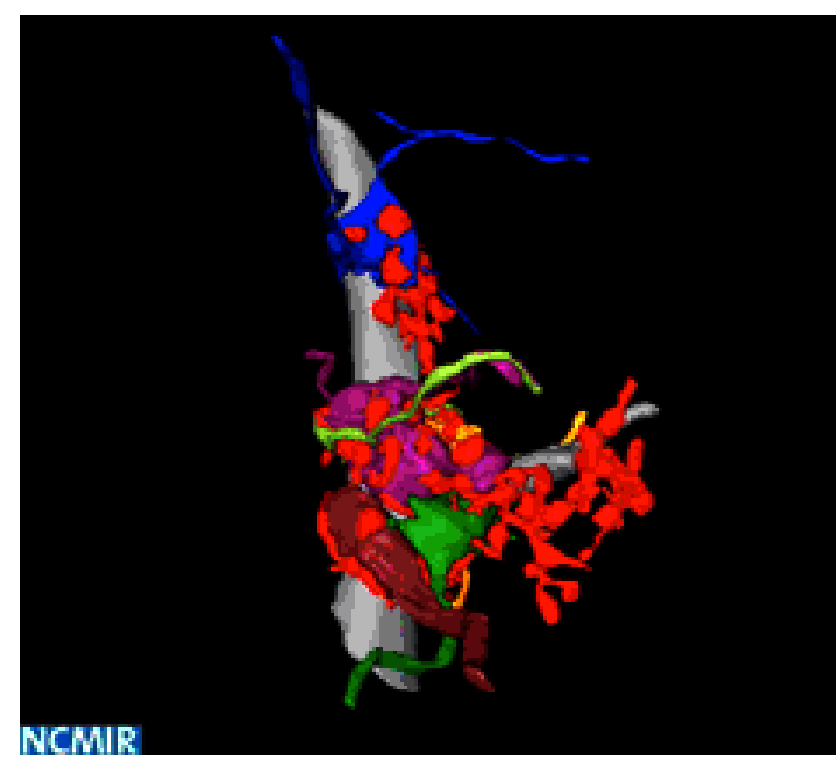

Movie 5. Example Adult SBEM reconstruction. CA3 dendritic shaft (silver) is shown with full complement of dendritic spines (initially shown in separate colors and then turn to red), followed by sequential appearance of six contacting MF axons/boutons (remaining colors). Not all MF inputs are shown.

tacts were significantly larger than their developing counterparts and occupied a much higher fraction of TE membrane surface area (Fig. 10E,F). Interestingly, immature TE spines had a greater number of distinct synaptic contacts per unit TE volume compared with mature TE spines (Fig. 10G). Thus, during the course of MF synaptic development, a larger number of small synapses is refined to a smaller number of larger synapses that may support more reliable synaptic transmission.

\section{Discussion}

The technical advances in SBEM described here allowed us to obtain a quantitative analysis of MF terminal development bridg- 


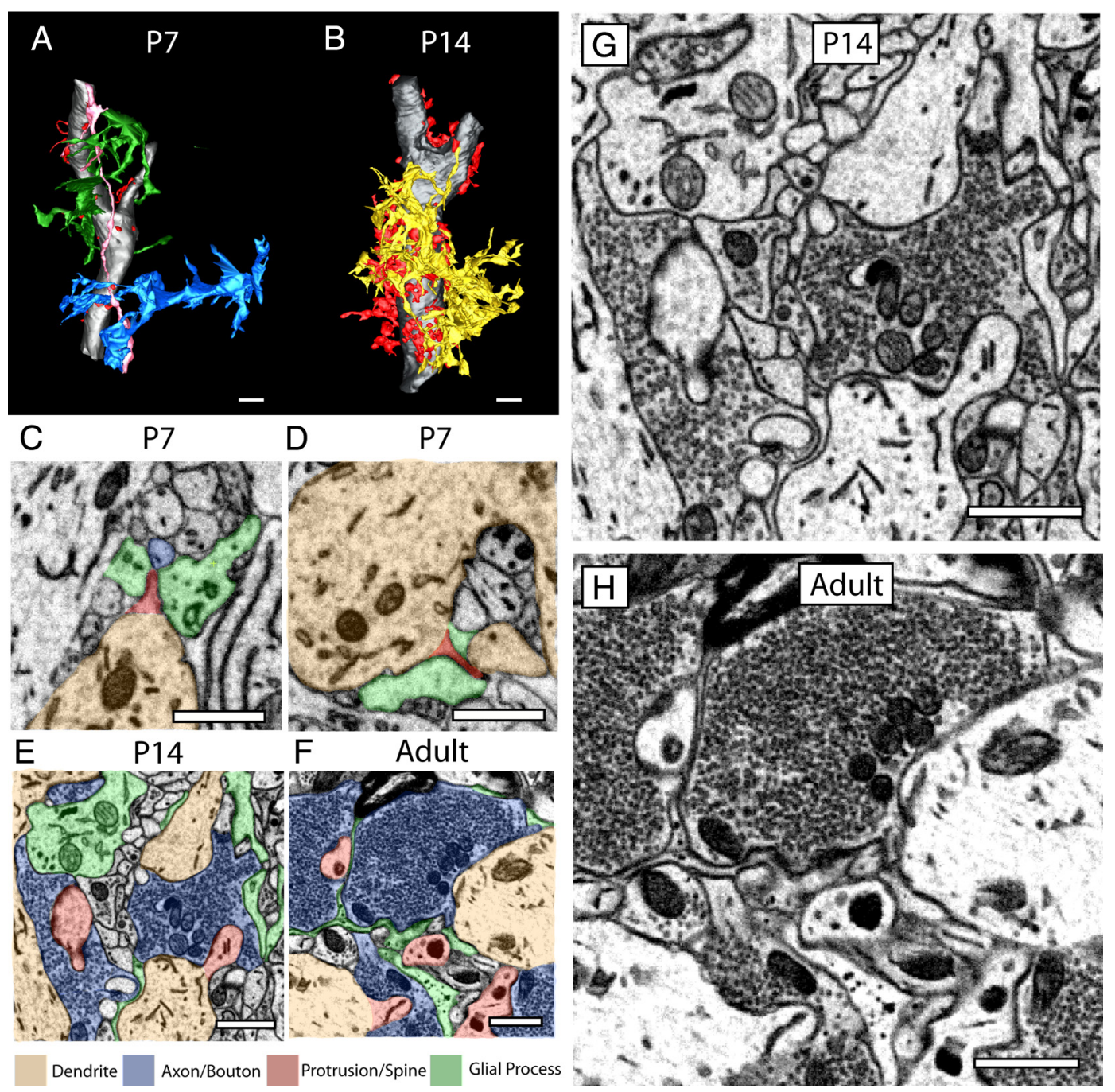

Figure 7. Glial cells contact sites of initial spine outgrowth. $A$, Dendritic shaft (silver) and spines (red) shown at P7 in close association with three separately reconstructed glial cell processes (blue, green, and pink). $\boldsymbol{B}, \mathrm{P} 14$ dendritic shaft (silver) and spines (red) shown in close association with a single large, partially reconstructed glial cell (yellow). $\boldsymbol{C}-\boldsymbol{F}$, EM cross-sections showing glia (green), dendritic shafts (orange), dendritic protrusions (red), and axonal structures (blue). C, P7: immature dendritic protrusion forming initial contact with undifferentiated axon, in close association with glial processes. D, P7: immature dendritic protrusion only in contact with glial processes. $\boldsymbol{E}, \mathrm{P} 14$ : glial processes in association with synaptic terminals. $\boldsymbol{F}$, Adult: glial processes surrounding synaptic terminals. $\boldsymbol{G}$, Enlarged micrograph from $\boldsymbol{E}$ without pseudocoloring. $\boldsymbol{H}$, Enlarged micrograph from $\boldsymbol{F}$ without pseudocoloring. Scale bars: $\boldsymbol{A}, \boldsymbol{B}, 2 \mu \mathrm{m} ; \boldsymbol{C}-\boldsymbol{H}, 1 \mu \mathrm{m}$.

ing spatial scales from synaptic ultrastructure to patterns of dendritic innervation that would be exceedingly difficult using standard methodologies. The MF terminal is large and complex, exhibiting significant morphological diversity relative to typical excitatory synapses in the neocortex. SBEM enabled us to capture this diversity through the complete reconstruction of a much larger number of MF terminals than in previous studies (Chicurel and Harris, 1992; Rollenhagen et al., 2007). Analysis across these scales is crucial not only for a complete understanding of the assembly of elements composing local microcircuits but also for obtaining accurate measurements of cellular parameters for biophysical models of circuit function.

The results achieved using this technique provide images of sufficient resolution to analyze synaptic connectivity using synaptic vesicles and PSDs, which was previously considered a limitation of SBEM. A recent study by Mishchenko et al. (2010) used an automated algorithm to fully reconstruct serial-section transmission EM (TEM) volumes totaling nearly $700 \mu \mathrm{m}^{3}$ in volume. Our standard reconstruction volumes were $12,500 \mu \mathrm{m}^{3}$, which could be further increased by tiled imaging and stitching together of adjacent volumes, up to $\sim 75,000 \mu \mathrm{m}^{3}$ (data not shown). Because we use manual reconstruction techniques, our dataset consists of individual neurons and their associated presynaptic elements. The integration of such automated reconstruction algorithms such as that described by Mishchenko et al. (2010) to the large datasets obtained by SBEM would greatly enhance the power of this approach and should eventually enable full reconstruction of mammalian neural circuits.

\section{Technological considerations}

There has been considerable debate on whether SBEM can provide the resolution necessary for examining synaptic connectivity. With the described fixation, staining, and visualization conditions, we were able to obtain high-resolution images that could be used to identify subcellular organelles and ultrastructural correlates of synaptic connectivity, such as synaptic vesicles and PSDs (Figs. 2E, F, 3, 10). Thus, SBEM technology is a useful bridge between light-level analysis and the detailed ultrastructure of TEM given its utility for both large-scale volumetric reconstruction and analysis of synaptic connectivity. 

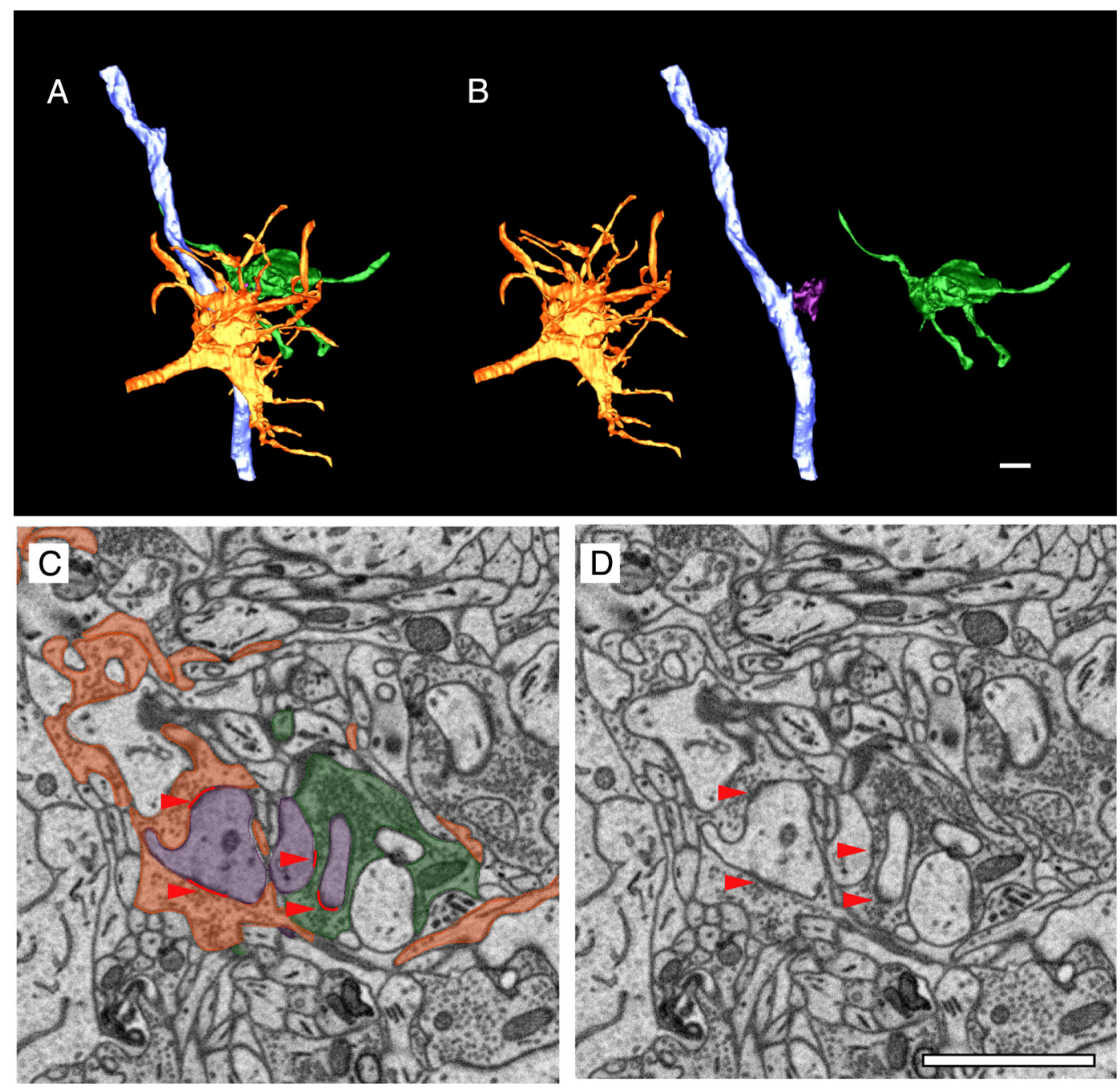

Figure 8. Single TE spines can receive synaptic contacts from multiple MFBs. $A$, Two reconstructed P14 MFBs (orange and green) shown in contact with a CA3 dendritic shaft (violet). For simplicity, only a single relevant TE spine is shown (purple), essentially obscured in $\boldsymbol{A}$ by the boutons. $\boldsymbol{B}$, Individual reconstructed elements from $\boldsymbol{A}$ shown separately. Single TE spine is clearly visible here (purple). C, Single cross-section of ultrastructural data from reconstruction with colors as indicated in $\boldsymbol{A}$. PSDs (red) at the interface between purple spine and each bouton are clearly visible and indicated with a red arrowhead. $\boldsymbol{D}$, Same ultrastructural cross-section as $\boldsymbol{E}$, without shading for a clearer view of ultrastructure. Scale bars, $2 \mu \mathrm{m}$.

Our analysis of SBEM data benefitted from a number of technical improvements that greatly improved the quality of the images. SBEM uses backscattered electrons to form images rather than transmitted electrons (Denk and Horstmann, 2004). Because all staining must be conducted en bloc, before sectioning, the samples must be stained more heavily than in traditional TEM. This staining with heavy metals is necessary to achieve sufficient membrane contrast but also to increase the conductivity of the sample and prevent the buildup of charge associated with electron bombardment. If charge is unable to dissipate, artifact is introduced and the lateral resolution of EM micrographs is compromised.

To the original osmium and uranyl acetate staining protocol developed by Denk and Horstmann (2004) for SBEM we added thiocarbohydrazide. Thiocarbohydrazide links osmium ions, allowing for an increased osmium concentration within the tissue, and has long been used to prepare specimens for scanning EM (Kelley et al., 1973). We used a combination of ferrocyanidereduced $\mathrm{OsO}_{4}$ and unreduced $\mathrm{OsO}_{4}$, a preparation that has been demonstrated to improve membrane staining (Hayat, 2000). Specimen charging was further reduced by electrically grounding the sample to the aluminum specimen pin with silver paint and a gold-palladium sputter coating. Gas in the microscope chamber can also act to dissipate charge but results in scatter of electrons and degradation of resolution. Because of the above measures to reduce specimen charging, we were usually able to image at high vacuum, which also resulted in enhanced image resolution.

\section{Insights into MF terminal and synapse development}

Our study provides evidence for early innervation of CA3 dendrites by DG MF axons. As early as P0 we observe evidence of target selection, with small collections of vesicles clustered at the sites of axon-dendrite contact. By P7, there are many more inputs, and these often have small axonal expansions, with vesicles clustered at the interface with the CA3 dendritic shaft. At P7, there are also increasing numbers of immature TE spines. It has been proposed that MFBs directly induce the formation of TE protrusions at CA3 neurons. This is based on the observation that MFBs were seen to precede spines in early development and on the fact that lesioning of the MF pathway in vitro causes a loss of spines, whereas reinnervation leads to their reappearance (Robain et al., 1994). We find significant development of CA3 dendritic protrusions at $\mathrm{P} 7$, earlier than previous reports (Amaral and Dent, 1981), and also that $>50 \%$ of immature protrusions 

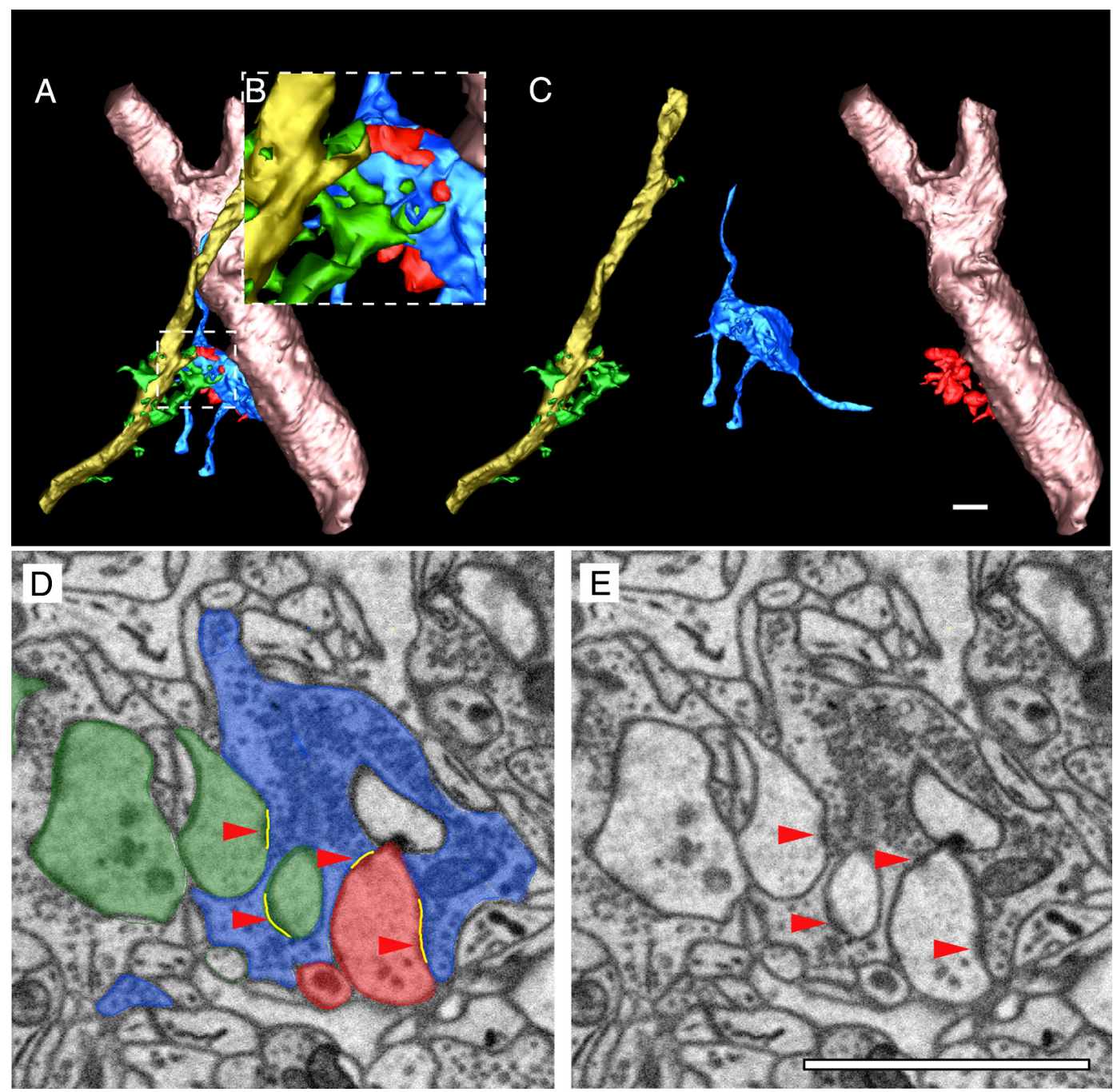

Figure 9. Single MFBs can contact TE spines on dendrites from two separate neurons. $\boldsymbol{A}$, Reconstructions of two separate P14 dendrites (olive with green spines and pink with red spines) are shown in contact with a single reconstructed presynaptic bouton (blue). Only a subset of relevant spines is shown for ease of interpretation. $\boldsymbol{B}$, Higher-magnification view of dashed box in $\boldsymbol{A}$. $\boldsymbol{C}$, Each individual element as in $\boldsymbol{A}$, shown separately. $\boldsymbol{D}$, Single cross-section of ultrastructural data used for this reconstruction, showing bouton (blue) and spines (green and red). PSDs (yellow) are clearly visible between the bouton and spines from each neuron as indicated by red arrowheads. $\boldsymbol{E}$, Same cross-section as in $\boldsymbol{E}$, without shading for a clearer view of ultrastructure. Scale bars, $2 \mu \mathrm{m}$.

exist in the absence of any axonal specialization (Fig. 6O). Previous studies may not have fully appreciated these small spine precursors as a result of the limitations of available methodologies. Therefore, our findings argue against a direct induction of protrusions by MF axonal contact but rather support a more active role of CA3 dendritic protrusions in synaptogenesis. That many unassociated spine precursors emerge at sites in which they are only contacted by glia is interesting given the increasing evidence for an active role of glial cells in synaptogenesis (for review, see Ullian et al., 2004).

The most striking changes in axonal and dendritic morphology occur during the second postnatal week. During this brief period, MFBs and TEs transition from relatively undifferentiated structures to their mature forms. MFBs differentiate from small axonal bulges into dramatic expansions sprouting many fine filopodia. Although some filopodial extensions made synaptic contact with interneurons as described previously (Acsády et al., 1998; Lawrence and McBain, 2003; Mori et al., 2004; McBain, 2008), most that were traced to their ends did not. Early in development, MFB filopodia are more motile than in mature circuits, and motility is inversely correlated with synaptic contact
(Tashiro et al., 2003). Similarly, filopodial processes of cerebellar MFs contact inappropriate synaptic partners early in development before eventually being eliminated (Kalinovsky et al., 2011). These findings support an exploratory function for immature MFB filopodia in either seeking out contact with interneuron targets or expanding the territory of the main MFB. In fact, many shorter MFB processes were observed to enter into the domains of other MFB terminals and contact spine heads (data not shown). This may reflect a competitive process by which MFBs seek to expand their territory at the expense of neighboring terminals. Increases in MFB filopodia number have been implicated recently in learning-related reorganization of MF microcircuitry (Ruediger et al., 2011). A similar, developmental phenomenon associated with circuit formation is supported by the striking refinement of presynaptic filopodia, with P14 MFBs having four times more filopodia than adult MFBs.

The capability to analyze the ultrastructural components of synapses, such as PSDs and synaptic vesicles, in conjunction with MF synapse morphological development is a major advantage of this technique. At $\mathrm{P} 0$ and $\mathrm{P} 7$, even when MFBs are in direct contact with dendritic protrusions, there is rarely evidence of 


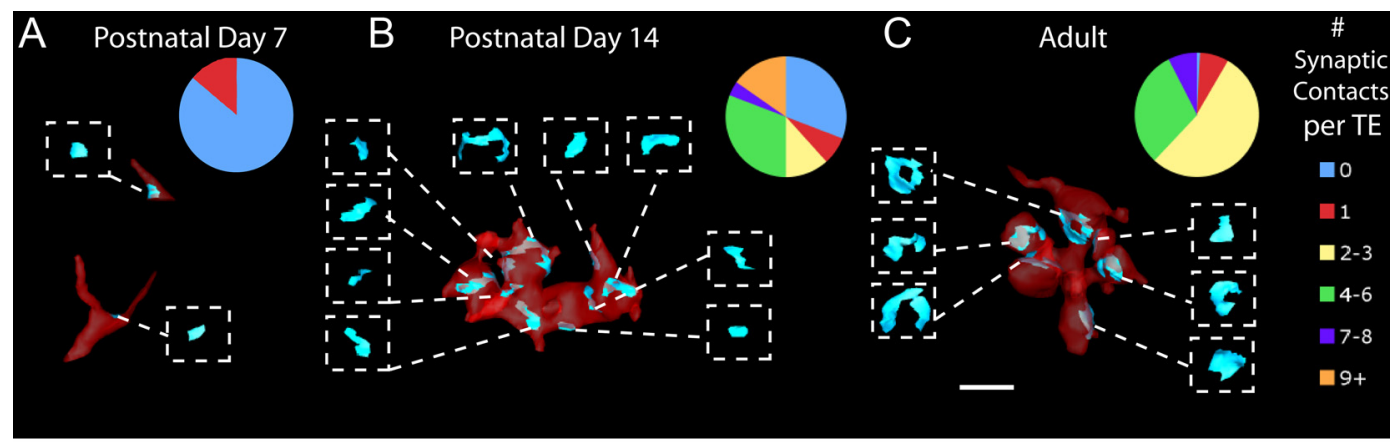

D

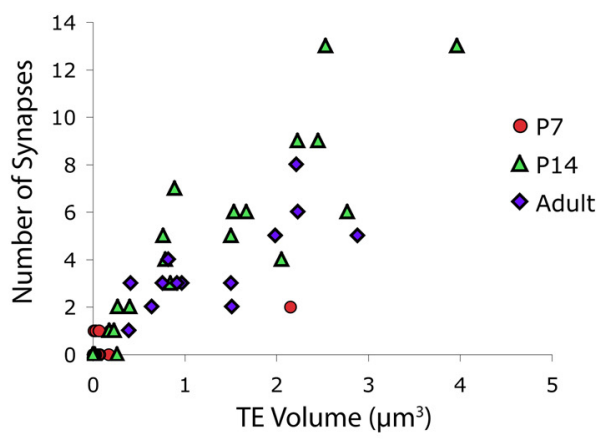

$\mathrm{F}$

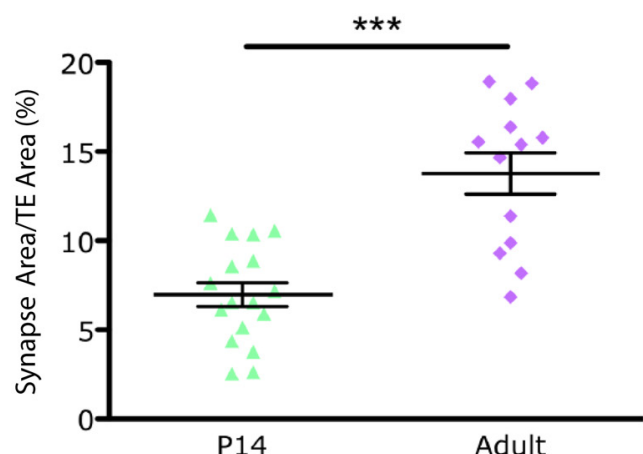

E

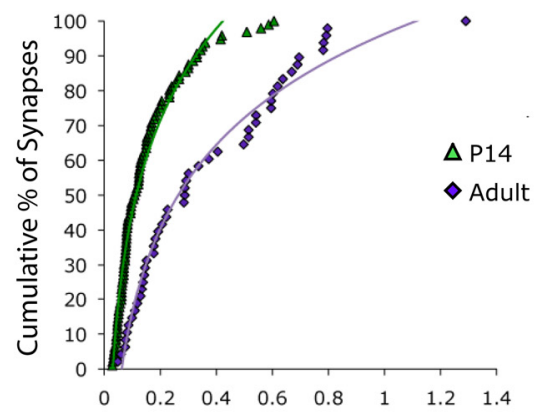

Synaptic Contact Surface Area $\left(\mu \mathrm{m}^{2}\right)$

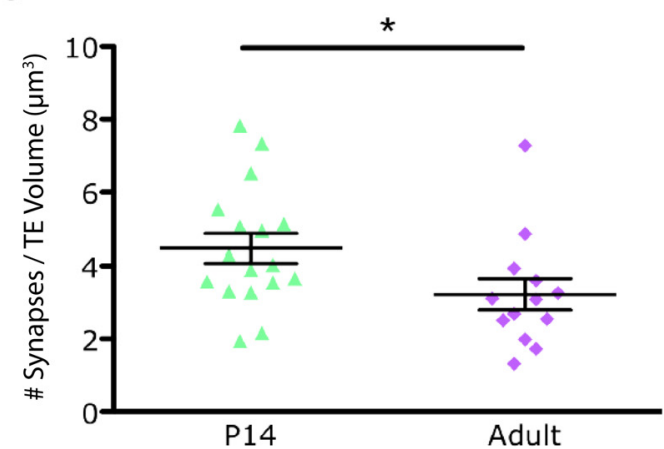

Figure 10. Refinement of MF-CA3 synaptic contacts during postnatal development. A-C, Example TE spines at P7, P14, and adult time points (red) with synaptic sites (blue) (inset represents each synaptic site viewed head on), the number of synaptic contacts per spine is represented in terms of relative frequency as a wedge diagram for each developmental stage. $\boldsymbol{D}$, Quantification of the number of synaptic sites relative to TE volume in cubic micrometers for P7, P14, and adult time points. E, Cumulative distribution plot of synaptic site surface areas in square micrometers for P14 and adult time points. $F$, Quantification of the percentage of TE surface area occupied by synaptic sites for P14 and adult time points. G, Scatter plot representation of the relationship between TE surface area and number of synaptic sites for P7, P14, and adult time points. Scale bar, $1 \mu \mathrm{m} .{ }^{*} p<0.05{ }^{* * *} p<0.001$, by $t$ test. Error bars represent \pm SEM.

synapses onto spine precursors. This finding suggests that MF synaptic currents elicited during this epoch are likely mediated by synaptic transmission directly onto dendritic shafts and represent an intermediate stage in functional and morphological maturation.

MF connectivity continues to evolve substantially between P14 and adult time points. It is interesting that morphological refinement of MFB structure and in MFB and TE association is matched by ultrastructural refinement of synaptic contacts during this extended period of maturation. It appears that a large number of smaller synaptic contacts in the immature state is refined to a smaller set of synaptic contacts, each encompassing a larger area in maturity (Fig. 10). This could be accomplished either by a competitive model in which some sites win out and enlarge to become dominant or perhaps initial small synaptic sites merge to become more powerful. Other mechanisms of refinement may also be at play. For example, at P14, we find that a single TE spine can receive inputs from multiple MFBs (Fig. 8) and a single MFB can contact TE spines from two separate den- drites of distinct CA3 neurons (Fig. 9). These may reflect transient interactions during circuit refinement, because they were not observed in the adult.

In conclusion, the observations reported here illustrate the power of SBEM to study the development of neural circuits. Using this approach, we are able to provide a greater level of detail and a more complete quantitative description of the full range of MF terminal and TE spine diversity compared with other techniques. By simultaneously investigating the development of axonal and dendritic specializations, we are able to gain insight into the mechanistic process by which the MF synapse is assembled. Finally, we identify several previously undescribed features of developmental refinement in MF terminal structure, including alterations in the organization of synaptic contacts and a refinement of MFB filopodia. SBEM represents an exciting technical advance, which when combined with the growing arsenal of tools for studying the nervous system in vivo has enormous potential to advance our understanding of the connectivity of neural circuits. 


\section{References}

Acsády L, Kamondi A, Sík A, Freund T, Buzsáki G (1998) GABAergic cells are the major postsynaptic targets of mossy fibers in the rat hippocampus. J Neurosci 18:3386-3403. Medline

Amaral DG, Dent JA (1981) Development of the mossy fibers of the dentate gyrus. I. A light and electron microscopic study of the mossy fibers and their expansions. J Comp Neurol 195:51-86. CrossRef Medline

Amaral DG, Ishizuka N, Claiborne B (1990) Neurons, numbers and the hippocampal network. Prog Brain Res 83:1-11. CrossRef Medline

Arellano JI, Benavides-Piccione R, Defelipe J, Yuste R (2007) Ultrastructure of dendritic spines: correlation between synaptic and spine morphologies. Front Neurosci 1:131-143. CrossRef Medline

Bischofberger J, Engel D, Frotscher M, Jonas P (2006) Timing and efficacy of transmitter release at mossy fiber synapses in the hippocampal network. Pflugers Arch 453:361-372. CrossRef Medline

Briggman KL, Denk W (2006) Towards neural circuit reconstruction with volume electron microscopy techniques. Curr Opin Neurobiol 16:562570. CrossRef Medline

Briggman KL, Helmstaedter M, Denk W (2011) Wiring specificity in the direction-selectivity circuit of the retina. Nature 471:183-188. CrossRef Medline

Chicurel ME, Harris KM (1992) Three-dimensional analysis of the structure and composition of CA3 branched dendritic spines and their synaptic relationships with mossy fiber boutons in the rat hippocampus. J Comp Neurol 325:169-182. CrossRef Medline

Dailey ME, Buchanan J, Bergles DE, Smith SJ (1994) Mossy fiber growth and synaptogenesis in rat hippocampal slices in vitro. J Neurosci 14:10601078. Medline

Denk W, Horstmann H (2004) Serial block-face scanning electron microscopy to reconstruct three-dimensional tissue nanostructure. PLoS Biol 2:329. CrossRef Medline

De Roo M, Klauser P, Mendez P, Poglia L, Muller D (2008) Activitydependent PSD formation and stabilization of newly formed spines in hippocampal slice cultures. Cereb Cortex 18:151-161. CrossRef Medline

Galimberti I, Gogolla N, Alberi S, Santos AF, Muller D, Caroni P (2006) Long-term rearrangements of hippocampal mossy fiber terminal connectivity in the adult regulated by experience. Neuron 50:749-763. CrossRef Medline

Harris KM, Stevens JK (1989) Dendritic spines of CAl pyramidal cells in the rat hippocampus: serial electron microscopy with reference to their biophysical characteristics. J Neurosci 9:2982-2997. Medline

Hayat MA (2000) Principles and techniques of electron microscopy: biological applications, Ed 4. Cambridge, UK: Cambridge UP.

Henze DA, McMahon DB, Harris KM, Barrionuevo G (2002a) Giant miniature EPSCs at the hippocampal mossy fiber to CA3 pyramidal cell synapse are monoquantal. J Neurophysiol 87:15-29. Medline

Henze DA, Wittner L, Buzsáki G (2002b) Single granule cells reliably discharge targets in the hippocampal CA3 network in vivo. Nat Neurosci 5:790-795. CrossRef Medline

Kalinovsky A, Boukhtouche F, Blazeski R, Bornmann C, Suzuki N, Mason CA, Scheiffele P (2011) Development of axon-target specificity of ponto-cerebellar afferents. PLoS Biol 9:e1001013. CrossRef Medline

Kelley RO, Dekker RA, Bluemink JG (1973) Ligand-mediated osmium binding: its application in coating biological specimens for scanning electron microscopy. J Ultrastruct Res 45:254-258. CrossRef Medline

Knott GW, Holtmaat A, Wilbrecht L, Welker E, Svoboda K (2006) Spine growth precedes synapse formation in the adult neocortex in vivo. Nat Neurosci 9:1117-1124. CrossRef Medline

Kremer JR, Mastronarde DN, McIntosh JR (1996) Computer visualization of three-dimensional image data using IMOD. J Struct Biol 116:71-76. CrossRef Medline

Lawrence JJ, McBain CJ (2003) Interneuron diversity series: containing the detonation-feedforward inhibition in the CA3 hippocampus. Trends Neurosci 26:631-640. CrossRef Medline

Leutgeb JK, Leutgeb S, Moser MB, Moser EI (2007) Pattern separation in the dentate gyrus and CA3 of the hippocampus. Science 315:961-966. CrossRef Medline

Marchal C, Mulle C (2004) Postnatal maturation of mossy fibre excitatory transmission in mouse CA3 pyramidal cells: a potential role for kainate receptors. J Physiol 561:27-37. CrossRef Medline

McBain CJ (2008) Differential mechanisms of transmission and plasticity at mossy fiber synapses. Prog Brain Res 169:225-240. CrossRef Medline

Mishchenko Y, Hu T, Spacek J, Mendenhall J, Harris KM, Chklovskii DB (2010) Ultrastructural analysis of hippocampal neuropil from the connectomics perspective. Neuron 67:1009-1020. CrossRef Medline

Mori M, Abegg MH, Gähwiler BH, Gerber U (2004) A frequencydependent switch from inhibition to excitation in a hippocampal unitary circuit. Nature 431:453-456. CrossRef Medline

Nicoll RA, Schmitz D (2005) Synaptic plasticity at hippocampal mossy fibre synapses. Nat Rev Neurosci 6:863-876. CrossRef Medline

Nimchinsky EA, Sabatini BL, Svoboda K (2002) Structure and function of dendritic spines. Annu Rev Physiol 64:313-353. CrossRef Medline

Robain O, Barbin G, Billette de Villemeur T, Jardin L, Jahchan T, Ben-Ari Y (1994) Development of mossy fiber synapses in hippocampal slice culture. Brain Res Dev Brain Res 80:244-250. CrossRef Medline

Rollenhagen A, Sätzler K, Rodríguez EP, Jonas P, Frotscher M, Lübke JH (2007) Structural determinants of transmission at large hippocampal mossy fiber synapses. J Neurosci 27:10434-10444. CrossRef Medline

Ruediger S, Vittori C, Bednarek E, Genoud C, Strata P, Sacchetti B, Caroni P (2011) Learning-related feedforward inhibitory connectivity growth required for memory precision. Nature 473:514-518. CrossRef Medline

Shepherd GM, Harris KM (1998) Three-dimensional structure and composition of CA3 $\rightarrow$ CA1 axons in rat hippocampal slices: implications for presynaptic connectivity and compartmentalization. J Neurosci 18:83008310. Medline

Sorra KE, Harris KM (2000) Overview on the structure, composition, function, development, and plasticity of hippocampal dendritic spines. Hippocampus 10:501-511. CrossRef Medline

Tashiro A, Dunaevsky A, Blazeski R, Mason CA, Yuste R (2003) Bidirectional regulation of hippocampal mossy fiber filopodial motility by kainite receptors: a two-step model of synaptogenesis. Neuron 38:773-784. CrossRef Medline

Toni N, Laplagne DA, Zhao C, Lombardi G, Ribak CE, Gage FH, Schinder AF (2008) Neurons born in the adult dentate gyrus form functional synapses with target cells. Nat Neurosci 11:901-907. CrossRef Medline

Ullian EM, Christopherson KS, Barres BA (2004) Role for glia in synaptogenesis. Glia 47:209-216. CrossRef Medline

Walton J (1979) Lead aspartate, an en bloc contrast stain particularly useful for ultrastructural enzymology. J Histochem Cytochem 27:1337-1342. CrossRef Medline

Ziv NE, Smith SJ (1996) Evidence for a role of dendritic filopodia in synaptogenesis and spine formation. Neuron 17:91-102. CrossRef Medline 